\title{
MODULAR OPERADS AND THE NERVE THEOREM
}

\author{
PHILIP HACKNEY, MARCY ROBERTSON, AND DONALD YAU
}

\begin{abstract}
We describe a category of undirected graphs which comes equipped with a faithful functor into the category of (colored) modular operads. The associated singular functor from modular operads to presheaves is fully faithful, and its essential image can be classified by a Segal condition. This theorem can be used to recover a related statement, due to André Joyal and Joachim Kock, concerning a larger category of undirected graphs whose functor to modular operads is not just faithful but also full.
\end{abstract}

The inclusion of the simplex category $\Delta$ into Cat, the category of small categories, induces a fully faithful functor from Cat into the category $\mathbf{S e t}^{\Delta^{\mathrm{op}}}$ of presheaves, via the assignment $\mathbf{C} \mapsto \operatorname{Fun}(-, \mathbf{C})$. It is classical that the essential image of this functor consists of those presheaves $X$ which satisfy a Segal condition; that is, for every $n \geq 2$ the set $X_{n}$ can be described as an iterated pullback

$$
X_{n} \cong \underbrace{X_{1} \times_{X_{0}} X_{1} \times_{X_{0}} \cdots \times_{X_{0}} X_{1}}_{n} .
$$

To goal of this paper is to extend this story to the setting of modular operads.

A modular operad GK98 is an algebraic structure consisting of a sequence of $\Sigma_{n}$-sets $P(n)$, indexed on nonnegative integers $n$, together with

- 'composition operations' $P(n) \times P(m) \rightarrow P(n+m-2)$, one for each pair of integers $(i, j) \in[1, n] \times[1, m]$ and

- 'contraction operations' $P(n) \rightarrow P(n-2)$, one for each pair of integers $(i, j)$ with $0 \leq i<j \leq n$.

This paper, along with its companion [HRY20], center around a new category of graphs that permit a Segalic approach to the study of modular operads. This category is a refined version (see Remark 1.8) of a category of graphs studied by André Joyal and Joachim Kock in JK11.

Our modular graphical category, called U, was developed in the companion paper HRY20. The objects of this category are undirected, connected graphs with loose ends, while morphisms are given by 'blowing up' vertices of the source into subgraphs of the target in a way that reflects iterated operations in a modular operad. Regarding a graph as a colored modular operad generated by its vertices, one should have a (faithful) functor $\mathbf{U} \rightarrow$ ModOp into the category of colored modular operads. Our main theorem, which reappears later as Theorem 3.6, is that (colored) modular operads can be characterized as certain objects in the category $\widehat{\mathbf{U}}=$ Set $^{\mathbf{U}^{\text {op }}}$ of $\mathbf{U}$-presheaves.

Date: April 26, 2020.

The first author acknowledges the support of Australian Research Council Discovery Project grant DP160101519. 
Theorem A. The functor $\mathbf{U} \rightarrow$ ModOp induces a fully-faithful functor $N$ : ModOp $\rightarrow \widehat{\mathbf{U}}$. The essential image of $N$ consists precisely of those presheaves which satisfy a strict Segal condition.

Part of the content of this theorem is the description of the functor from graphs to modular operads. In this paper, the color set of a modular operad is actually an involutive color set, where color matching for composition and contraction operations are governed by the involution (similar to the situation for cyclic multicategories in CGR14]). For example, given a graph $G$, the associated modular operad has color set of cardinality $2|E(G)|$, with one color for each possible orientation on each edge. If $e$ is an edge of $G$ joining two vertices $v$ and $w$, the generating operations $v$ and $w$ will be tagged with opposing orientations of the edge $e$, and so can be formally composed.

Modular operads as we define them here were first introduced (for the ground category Set) in JK11, where they are called 'compact symmetric multicategories.' These were further studied in the thesis of Sophie Raynor Ray18 and in Ray19. Most geometric examples of colored modular operads in the literature have been in the setting where the involution on color sets is trivial, as in Gia13, [HVZ10, and [KW17]. A notable exception is [Pet13, which had a class of examples which were colored by involutive groupoids, rather than involutive sets. On the other hand, Drummond-Cole and the first author studied colored cyclic operads with involutive set of colors in DCH18. Working with involutive color sets had distinct homotopical advantages in that work, which were already clear in [DCH19, 2.11]. But it had a further advantage: colored cyclic operads (in the sense of [HRY19]), colored operads, and colored dioperads can all be considered as special types of colored cyclic operads when allowing for involutive color sets. Likewise, our more general notion of modular operad that we consider in this paper allows one to regard wheeled properads as a special case.

The category $\mathbf{U}$ from the above is a subcategory of ModOp, but it is not a full subcategory. Instead, it is generated by morphisms that are local in nature, involving two or fewer vertices. In the companion paper (see also Remark 1.8), this restriction is used to show that $\mathbf{U}$ admits a generalized Reedy structure [BM11] (allowing us to use the Reedy model structure on categories of diagrams), which may not be true for the full subcategory $\mathbf{G r}$ of ModOp spanned by the graphs. The second theorem of our paper (appearing later as Theorem 4.1), is the following.

Theorem B (Joyal-Kock 2011). The full subcategory inclusion $\mathbf{G r} \rightarrow$ ModOp induces a fully-faithful functor $N_{J K}: \mathbf{M o d O p} \rightarrow \widehat{\mathbf{G r}}$. The essential image of $N_{J K}$ consists precisely of those presheaves which satisfy a strict Segal condition.

This theorem was announced in [JK11, and in Section 4 we show how this follows from Theorem A. This is the first publicly available proof of Theorem B Our proof does not use the techniques proposed by Joyal and Kock.

Related work. The topic of nerve theorems has a rich literature (that we cannot hope to cover adequately), including a general machine [BMW12, Web07] that one can use to prove nerve theorems. This was used by Weber Web07 to prove a nerve theorem for operads involving the dendroidal category $\Omega$ from MW07. (see also the later account [Koc11, Theorem 2.5.4]). This is also the approach towards Theorem B that was indicated in [JK11]. In her thesis Ray18], Sophie Raynor 
proved two variations of Theorem $\mathrm{B}$ along these lines: one dealt with non-unital modular operads, while the unital version used an alternative category of graphs (see also Ray19]). In contrast, Theorem A does not fit into the framework of [BMW12, as $\mathbf{U}$ is not a full subcategory of ModOp. Instead, the situation is more akin to the approach to the dendroidal nerve theorem found in the work of Cisinski, Moerdijk, and Weiss (see, for instance, CM13, Corollary 2.6]).

Further directions. In [HRY20 we explained the notion of (inner and outer) coface maps of $\mathbf{U}$. Given a coface map $\delta$ with codomain $G$, one can define the horn $\Lambda^{\delta}[G]$ which is a subobject of the representable object $\mathrm{U}[G]$. A strict inner Kan presheaf $X$ is a presheaf such that every diagram with $\delta$ an inner coface map

$$
\begin{array}{ccc}
\Lambda^{\delta}[G] & \rightarrow & \longrightarrow \\
\downarrow & \exists ! \\
\cup[G] &
\end{array}
$$

admits a unique filler. One could ask if the presheaves of Theorem A coincide with the strict inner Kan presheaves, in analogy with the situation for categories, operads [MW09], properads [HRY15], and so on. See also Remark 2.16.

Outline. We begin the paper by recalling, in Section 1, essential information about $\mathbf{U}$ from the companion paper [HRY20. Section 2 deals with modular operads, and is split into two subsections, the first of which gives a monadic definition of modular operad valid in any closed symmetric monoidal category E. As we are working with modular operads with involutive color sets, this definition is technically new, but we regard this section as background. The heart of the paper begins in 22.2 , where we construct, for each graph $G$, a modular operad $\langle G\rangle$. This is part of a functor $J: \mathbf{U} \rightarrow$ ModOp, and in Section 3 we use this functor to prove Theorem A The most delicate part is found in 3.1 , where we associate a modular operad to any Segal U-presheaf. The final section indicates how to recover Theorem $\mathrm{B}$ from Theorem A

Acknowledgments. This paper owes a lot to discussions several years ago with both André Joyal and Joachim Kock. We are also grateful to Sophie Raynor for explaining her thesis work to us. Finally, we'd like to thank various members of the Centre of Australian Category Theory for their questions and suggestions as this project developed.

Notation. Let $\mathbf{C}$ be a category. If $x$ and $y$ are objects of $\mathbf{C}$, we will write either $\operatorname{hom}(x, y)$ or $\mathbf{C}(x, y)$ for the set of morphisms from $x$ to $y$. We will write $\widehat{\mathbf{C}}$ for the category of $\mathbf{C}$-presheaves, that is, contravariant functors from $\mathbf{C}$ to the category Set of sets.

\section{BACKGROUND ON THE GRAPHICAL CATEGORY U}

All material from this section appears in some form in the companion paper HRY20, where proofs and further details may be found. Here we've only included the essential topics needed to understand what follows.

Graphs in this paper are undirected and are allowed to have 'loose ends'; that is, it is not necessary for both ends (or either end) of an edge to touch a vertex. See Figure 1 for a picture of one such graph. A model to keep in mind (compare [JS91, §2]) is that a graph can be taken to be a pair $(X, V)$ where $X$ is a space, 


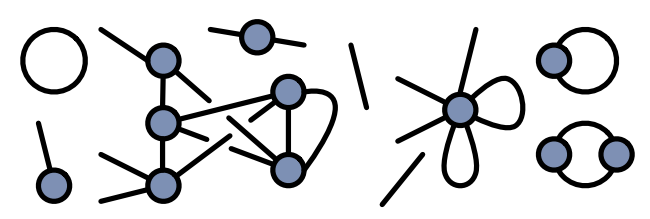

Figure 1. A typical graph with loose ends

$V$ is a finite set of points of $X$, and $X \backslash V$ is a one-manifold (without boundary) having only a finite set of connected components. Components of $X \backslash V$ are the edges of the graph, and elements of $V$ are the vertices. Thus we may have loops divorced from any vertex (those components of $X \backslash V$ homeomorphic to $S^{1}$ ), edges loose at one end (those with one missing limit point in $X$ ), and free floating edges (components of $X$ homeomorphic to $(0,1)$ which contain no vertices).

Such pictures lead to the following definition. The involutive set $A$ is the set of arcs, which are edges together with an orientation, and the involution $i$ swaps orientation. The partially-defined function $t: A \nrightarrow V$ takes an arc to the vertex it points towards.

Definition 1.1. A graph $G$ consists of

(a) a diagram of finite set: $\mathbb{1}^{1}$

$$
i \subset A \stackrel{s}{\longleftarrow} D \stackrel{t}{\longrightarrow} V
$$

and

(b) a subset $ð(G) \subseteq A \backslash s D$ called the boundary of $G$ so that

(A) $i$ is a fixedpoint-free involution,

(B) $s$ is a monomorphism,

(C) $i s D \backslash s D \subseteq \partial(G)$, and

(D) ठ(G) $\backslash i s D$ is an $i$-closed subset of $A$.

We will nearly always consider $D$ as a subset of $A$, and suppress the natural inclusion function $s: D \subseteq A$ from the notation. A graph will be called safe if $\precsim(G)=A \backslash s D$, while if the containment from (b) is strict then the graph will be called unsafe.

This definition is a modification of that in [JK11] in that it has a specified notion of boundary. There are several other combinatorial definitions of graphs BB17, JS91, YJ15] all of which are equivalent (Proposition 15.2, Proposition 15.6, and Proposition 15.8 of [BB17]) to this one.

Example 1.2 (Exceptional edge and nodeless loop). If $Z$ is a set, write $2 Z$ for the set

$$
\left\{z, z^{\dagger} \mid z \in Z\right\} \cong Z \amalg Z
$$

together with the evident involution. We consider $Z$ as a subset of $2 Z$, and write $Z^{\dagger}$ for its complement.

\footnotetext{
${ }^{1}$ To ensure that we have a set of graphs, insist that all of the sets $A, D, V$ are taken to be subsets of some fixed infinite set.
} 
- The exceptional edge, $\uparrow$, is the safe graph with $A=2\{*\}=ð(\uparrow)$ and $V=D=\varnothing$. As this graph is so important, we give special names to its arcs and write $A=\{\sharp, b\}$.

- A variation is to take $A=2\{*\}, V=D=\varnothing$, and have an empty boundary. We call graphs isomorphic to this one nodeless loops.

Recall that the neighborhood of a vertex $v \in V$ is defined to be $\operatorname{nb}(v)=t^{-1}(v) \subseteq$ $D$. The valence of a vertex $v$ is just the cardinality of the set $\operatorname{nb}(v)$.

Many other examples of graphs are given in the companion paper HRY20. For instance, if $G$ is connected and every vertex is bivalent, then $G$ is either a linear graph or a cycle.

Definition 1.3 (Stars). For $n \geq 0$, the $n$-star is $n$ has $V$ a one-point set, $D=$ $\{1, \ldots, n\}$, and $A=2 D$ (where 2(-) is as in Example 1.2). The function $s: D \rightarrow$ $A=2 D$ is just the subset inclusion. More generally, if $S$ is any set we define $\{S$ to be the (connected) graph with a single vertex so that $A=2 S, D=S^{\dagger} \subseteq 2 S$, and $\partial\left(\mho_{S}\right)=S \subseteq 2 S$. There are also variations of stars built from a fixed (connected) graph $G$.

- Let $\aleph_{G}$ be the one-vertex graph with $A=2 ð(G)$ and $D=ð(G)^{\dagger}$. Notice that we must have $\partial\left(\xi_{G}\right)=A \backslash D=\partial(G)$ and that the neighborhood of

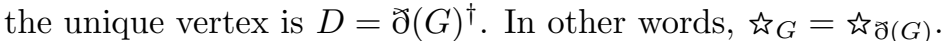

- Suppose that $v$ is a vertex of $G$ and let $\operatorname{nb}(v)$ be its neighborhood in $G$. We let $\boldsymbol{s}_{v}$ denote the graph with $V=\{v\}, D=\operatorname{nb}(v)$, and $A=2 \mathrm{nb}(v)$. The boundary of $\xi_{v}$ is $\operatorname{nb}(v)^{\dagger} \subseteq 2 \mathrm{nb}(v)$. There is a canonical embedding

$$
\iota_{v}: i_{v} \rightarrow G
$$

coming from the natural inclusions $\{v\} \subseteq V(G)$ and $\operatorname{nb}(v) \subseteq D(G)$.

Let us now recall several varieties of morphisms from [HRY20, and [JK11.

Definition 1.4 (Natural transformations of graphs). Let $\mathscr{I}$ denote the category with three objects and three generating arrows, of shape $\subset \bullet \leftarrow \bullet \rightarrow \bullet$. Part of the data of each graph $G$ is a functor from $\mathscr{I}$ into finite sets where the leftward arrow is sent to a monomorphism and the generating endomorphism is sent to a free involution.

- A graph is connected if this functor is connected as an object in FinSet ${ }^{\mathscr{I}}$ (that is, if it is nonempty and cannot be written as a nontrivial coproduct in this category).

- If $G$ and $G^{\prime}$ are graphs, then a natural transformation $G \rightarrow G^{\prime}$ is said to be étale if

(1) the right-hand square of

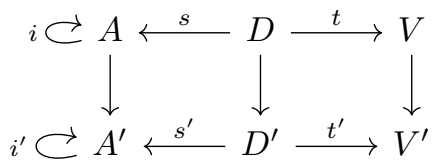

is a pullback, and

(2) the set $A \backslash(ð(G) \amalg D)$ maps into $A^{\prime} \backslash\left(ð\left(G^{\prime}\right) \amalg D^{\prime}\right)$.

- If $G$ and $G^{\prime}$ are connected graphs, then an étale map is called an embedding if $V \rightarrow V^{\prime}$ is a monomorphism. 
- The set $\widetilde{\operatorname{Emb}}(G)$ consists of all embeddings with codomain $G$. The set $\operatorname{Emb}(G)$ is the quotient of $\widetilde{\operatorname{Emb}}(G)$ by the relation that $f \sim h$ whenever there is an isomorphism $z$ with $f=h z$.

Note that (2) is automatically satisfied when $G$ is safe. The original definition of étale, from [JK11] only had condition (1) as all graphs were implicitly regarded as safe.

In order to state our definition of graphical map from HRY20, we need two supplementary definitions. Both of these are initially functions on $\widetilde{\operatorname{Emb}}(G)$, but as we saw in the companion paper these descend to $\operatorname{Emb}(G)$.

Definition 1.5 (Invariants of embeddings). Suppose that $G$ and $G^{\prime}$ are two (potentially unsafe) graphs.

- Given any étale map $f: G^{\prime} \rightarrow G$, there is a corresponding element

$$
\sum_{v \in V^{\prime}} f(v) \in \mathbb{N} V
$$

in the free commutative monoid on $V$. The vertex sum, denoted $\varsigma$ : $\operatorname{Emb}(G) \rightarrow \mathbb{N} V$, is the function that takes $\left[f: G^{\prime} \rightarrow G\right]$ to $\sum_{v \in V^{\prime}} f(v)$. As we restrict to embeddings, this function factors through the power set $\wp(V) \subseteq \mathbb{N} V$.

- The restriction of any embedding $f: G^{\prime} \rightarrow G$ to the boundary $\partial\left(G^{\prime}\right)$ is a monomorphism. We write $\partial: \operatorname{Emb}(G) \rightarrow \wp(A(G))$ for the function which takes $\left[f: G^{\prime} \rightarrow G\right]$ to $f\left(ð\left(G^{\prime}\right)\right) \subseteq A(G)$.

Definition 1.6 (Graphical category). The graphical category $\mathbf{U}$ has objects the safe, connected graphs. A morphism $\varphi: G \rightarrow G^{\prime}$ (where $G$ and $G^{\prime}$ are safe) consists of the following data:

- A map of involutive sets $\varphi_{0}: A \rightarrow A^{\prime}$

- A function $\varphi_{1}: V \rightarrow \operatorname{Emb}\left(G^{\prime}\right)$

These data should satisfy two conditions.

(i) The inequality $\sum_{v \in V} \varsigma\left(\varphi_{1}(v)\right) \leq \sum_{w \in V^{\prime}} w$ holds in $\mathbb{N} V^{\prime}$.

(ii) For each $v$, we have a (necessarily unique) bijection making the diagram

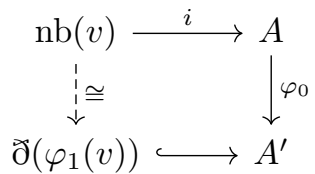

commute, where the top map $i$ is the restriction of the involution on $A$.

(iii) If the boundary of $G$ is empty, then there exists a $v$ so that $\varphi_{1}(v)$ is not an edge.

The extended graphical category $\widetilde{\mathbf{U}}$ is defined similarly, except the objects are allowed to be arbitrary connected graphs and condition (iii) for morphisms is replaced by

(iii') If the boundary of $G$ is empty and $\varphi_{1}(v)$ is an edge for every $v$, then $G^{\prime}$ is a nodeless loop.

The composition in $\mathbf{U}$ and $\widetilde{\mathbf{U}}$ are given by graph substitution. Let us recall the idea; a precise definition in our setting appears in Definition 1.10 Suppose that we are given a graph $G$, a collection of graphs $H_{v}$ indexed by the vertices of $G$, and 
specified bijections $i \operatorname{nb}(v) \cong ð\left(H_{v}\right)$. Then we can form a new graph $G\left\{H_{v}\right\}$ where we replace each vertex $v$ by the graph $H_{v}$, identifying the edges at the boundary of $H_{v}$ with the edges incident to the vertex $v$ in $G$.

Definition 1.7 (Composition of graphical maps). Suppose that $\varphi: G \rightarrow G^{\prime}$ and $\psi: G^{\prime} \rightarrow G^{\prime \prime}$ are graphical maps. We will define the composite $\psi \circ \varphi$. First, we have $(\psi \circ \varphi)_{0}=\psi_{0} \circ \varphi_{0}$. To define $(\psi \circ \varphi)_{1}(v)$, where $v$ is a vertex of $G$, first let $\varphi_{v}: K_{v} \hookrightarrow G^{\prime}$ be an embedding representing $\varphi_{1}(v)$. For a vertex $w$ in $\varphi_{v}\left(V\left(K_{v}\right)\right) \subseteq V\left(G^{\prime}\right)$, we can find an embedding $\psi_{w}: H_{w} \hookrightarrow G^{\prime \prime}$ representing $\psi_{1}(w)$. It turns out that the $\psi_{w}$ assemble into a single embedding ${ }^{2}$

$$
K_{v}\left\{H_{w}\right\} \hookrightarrow G^{\prime \prime}
$$

which factors each of the embeddings $\psi_{w}$. The function $(\psi \circ \varphi)_{1}$ sends $v$ to the class of (1) in $\operatorname{Emb}\left(G^{\prime \prime}\right)$.

See HRY20 for further details.

Remark 1.8. There is a related notion of morphism of connected graphs in JK11, but based on étale maps between connected safe graphs, rather than embeddings. Joyal and Kock do not include the conditions (ii) and (iii) of Definition 1.6 in their definition. Further, condition (ii) is modified to reflect that étale maps need not be injective on boundaries. This yields a category of connected safe graphs Gr, and each graphical map in the sense of Definition 1.6 is a morphism in Gr. The weak factorization system that is meant to exist on the category of Joyal and Kock becomes an orthogonal factorization system on our category ${ }_{3}^{3}$ which is much easier to work with. Moreover, our category admits a generalized Reedy structure in the sense of BM11, allowing us flexibility when considering model structures in the companion paper HRY20.

Embeddings constitute the right class of an orthogonal factorization system on $\mathbf{U}$ (resp. on $\widetilde{\mathbf{U}}$ ). Morphisms in the left class are called active maps.

Definition 1.9 (Active maps). A morphism $\varphi: G \rightarrow G^{\prime}$ is called active if $\varphi_{0}$ : $A \rightarrow A^{\prime}$ induces a bijection $\precsim(G) \rightarrow ð\left(G^{\prime}\right)$.

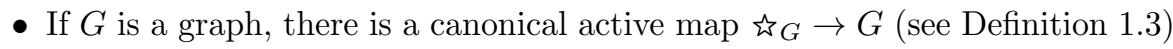
which sends the unique vertex of $\xi_{G}$ to $\left[\operatorname{id}_{G}: G \rightarrow G\right]$ and on arcs gives the identity on $ð\left(\aleph_{G}\right)=\succsim(G)$.

- More generally, if $G$ is a graph, $S$ is a set, and $\xi: S \rightarrow \partial(G)$ is a function, then there is an associated active map i $S_{S} \rightarrow G$ whose map on arcs restricts to $\xi: S \rightarrow ð(G) \subseteq A(G)$.

Before Definition 1.7, we mentioned the idea of graph substitution. In Construction 2.8, it will be helpful to have a concrete model on hand. Further, the notion of the Segal core of a graph is essential throughout this paper. As these concepts are closely related, we combine them into a single definition. Recall that if $G$ is a graph then the representable presheaf $\mathrm{U}[G]$ is the contravariant functor from $\mathbf{U}$ to Set with $\mathrm{U}[G]_{H}=\mathbf{U}(H, G)$.

\footnotetext{
${ }^{2}$ The fact that this is an embedding and not merely étale follows from (i) of Definition 1.6

${ }^{3}$ Compare with [Koc16, 2.4.14] in the directed setting, which is much simpler as embeddings in that context are monomorphisms.
} 
Definition 1.10 (Graph substitution and Segal cores). Suppose that $G$ is a connected graph containing at least one vertex, and let $E_{i}$ be its set of internal edges. For each internal edge $e \in E_{i}$, choose an ordering $e=\left[x_{e}^{1}, x_{e}^{2}\right]$ for the two-element equivalence class of arcs comprising $e$. The underlying functor of $G$ (in the diagram

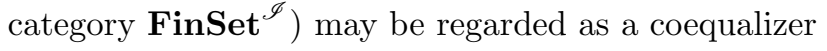

$$
\coprod_{e \in E_{i}} \uparrow \stackrel{\Re}{\underset{\Im}{\longrightarrow}} \coprod_{v \in V} \aleph_{v} \longrightarrow G
$$

where the map on the right is $\coprod_{v} \iota_{v}$. Explicitly, we have

- $\Re$ is the coproduct of maps $\Re_{e}: \uparrow \rightarrow \xi t x_{e}^{1}$ with $\Re_{e}(\sharp)=\left(x_{e}^{1}\right)^{\dagger} \in \partial\left(\xi t x_{e}^{1}\right)$ and $\Re_{e}(b)=x_{e}^{1} \in D\left(\aleph_{t x_{e}^{1}}\right)$;

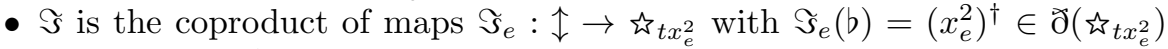
and $\Im_{e}(\sharp)=x_{e}^{2} \in D\left(\right.$ 羟 $\left.x_{e}^{2}\right)$.

- We first describe graph substitution. Suppose we are given graphs $H_{v}$ and isomorphisms $m_{v}$ from $i(\operatorname{nb}(v)) \subseteq A(G)$ to $\check{\partial}\left(H_{v}\right)$. We then have induced maps $\tilde{\Re}$ and $\widetilde{\Im}$, where

- $\tilde{\Re}$ is the coproduct of maps $\tilde{\Re}_{e}: \mathfrak{\imath} \rightarrow H_{t x_{e}^{1}}$ with $\tilde{\Re}_{e}(\sharp)=m_{t x_{e}^{1}}\left(i x_{e}^{1}\right) \in ð\left(H_{t x_{e}^{1}}\right)$,

- $\widetilde{\Im}$ is the coproduct of maps $\tilde{\Im}_{e}: \uparrow \rightarrow H_{t x_{e}^{2}}$ with $\tilde{\Im}_{e}(b)=m_{t x_{e}^{2}}\left(i x_{e}^{2}\right) \in \partial\left(H_{t x_{e}^{2}}\right)$. We can then form the coequalizer

$$
\coprod_{e \in E_{i}} \uparrow \underset{\tilde{\Re}}{\stackrel{\tilde{\Im}}{\longrightarrow}} \coprod_{v \in V} H_{v} \stackrel{\pi}{\longrightarrow} K .
$$

There is an induced monomorphism (see [HRY20]) $)(G) \rightarrow A(K) \backslash D(K)$ and we declare $ð(K)$ to be the image of this function. We write $G\left\{H_{v}\right\}$ for this graph, called graph substitution of $H_{v}$ into $G$.

- We likewise can form corresponding coequalizer to (2) in $\widehat{\mathbf{U}}$,

$$
\coprod_{e \in E_{i}} \mathrm{U}[\uparrow] \stackrel{\Re}{\underset{\Im}{\leftrightarrows}} \coprod_{v \in V} \mathrm{U}\left[\xi_{v}\right] \longrightarrow \mathrm{Sc}[G],
$$

and we call the target the Segal core of $G$. It comes with a map $\mathrm{Sc}[G] \rightarrow \mathrm{U}[G]$ induced by $\iota_{v}: \coprod_{v \in V} \mathrm{U}\left[\xi_{v}\right] \rightarrow \mathrm{U}[G]$. In the case when $G=\uparrow$, we declare the map $\mathrm{Sc}[G] \rightarrow \mathrm{U}[G]$ to be the identity map on $\mathrm{U}[G]$.

We return to Segal core definitions in a different context in Notation 3.11 .

\section{Modular operads}

In this section, we define (colored) modular operads in a closed monoidal category (\$2.1) and fabricate a class of examples coming from graphs (\$2.2). Our modular operads come equipped with an involution on color sets, and are an enriched version of the compact symmetric multicategories introduced in [JK11. All of the examples in 2.2 in fact come equipped with free involutions on the sets of colors.

Remark 2.1. At first glance it may appear that 2.1 depends on our particular choice of graph formalism (Definition 1.1). In fact, our constructions are relatively formalism agnostic, as long as we can get a handle on what the set of arcs (and the involution on that set) of a graph should be. For example, if one chooses to use Yau-Johnson graphs as in [YJ15, §1.2], then the set of arcs $A$ may be identified with $\operatorname{Flag}(G) \amalg \operatorname{Legs}_{\mathrm{o}}(G)$. The involution on $A$ is uniquely specified so that it 
- acts on this added $\operatorname{Legs}_{\mathrm{o}}(G)$ component by including into $\operatorname{Flag}(G)$,

- acts on $\operatorname{Flag}(G) \backslash \operatorname{Legs}(G)$ via $\iota_{G}$,

- acts on $\operatorname{Legs}_{\mathrm{e}}(G) \subseteq \operatorname{Flag}(G)$ by $\pi_{G}$.

We consider the subset $\operatorname{Legs}_{\mathrm{o}}(G) \subseteq \operatorname{Flag}(G)$ as being part of neighborhoods for some vertices, while the new summand $\operatorname{Legs}_{\mathrm{o}}(G)$ constitutes part of the boundary $ð(G)$. Specifically, $\partial(G)$ is the sum of this added $\operatorname{Legs}_{\mathrm{o}}(G)$ and $\operatorname{Legs}_{\mathrm{e}}(G) \subseteq \operatorname{Flag}(G)$.

Throughout this section 'graph' will mean 'connected graph' unless otherwise indicated. We emphasize that we are generally including nodeless loops as well, which is important in order to avoid the issue mentioned in Remark 2.15.

2.1. Monads governing modular operads. Let us fix a cocomplete, closed, symmetric monoidal category $(\mathbf{E}, \otimes, 1)$. In this subsection we give a monadic description of $\mathfrak{C}$-colored modular operads in $\mathbf{E}$, where $\mathfrak{C}$ is an involutive set. The monad in question is an adaptation of other existing monads for modular operads ([Mar08, §7], GK98, 2.17], [BB17, §10.1]) and generalized operadic structures ([BB17, §6], YJ15, 10.2,10.3]). It is also closely related to the monad from [JK11, $\S 5]$; see Remark 2.15. As such, the chief aim of the beginning of this subsection is to fix notation and provide enough background for the remainder of the paper. In Definition 2.13 we explain how to define morphisms between modular operads with different color sets.

Definition 2.2. Let $\mathbf{B}_{\mathfrak{C}}$ denote the groupoid with:

- objects pairs $(S, \xi)$, where $S$ is a finite set and $\xi: S \rightarrow \mathfrak{C}$ is a function, and - morphisms $(S, \xi) \rightarrow\left(S^{\prime}, \xi^{\prime}\right)$ are bijections $f: S \rightarrow S^{\prime}$ so that $\xi=\xi^{\prime} \circ f$.

In particular, $\mathbf{B}_{\{*\}}$ is just the usual category of finite sets and bijections. Note that Definition 2.2 ignores the involution present on the set $\mathfrak{C}$.

Remark 2.3. We could instead restrict this definition to the finite sets $\{1, \ldots, n\}$. In this case, a coloring function $\xi$ is the same thing as an ordered list $c_{1}, \ldots, c_{n}$ of elements of $\mathfrak{C}$. Suppose $\sigma$ is an automorphism of $\{1, \ldots, n\}$, considered as a morphism of $\mathbf{B}_{\mathfrak{C}}$ from $\xi \rightarrow \xi \sigma^{-1}$. Using the identification of $\xi$ with the list $c_{1}, \ldots, c_{n}$ and likewise for $\xi \sigma^{-1}$, the morphism $\sigma$ goes from $c_{1}, \ldots, c_{n}$ to $c_{\sigma^{-1}(1)}, \ldots, c_{\sigma^{-1}(n)}$. Thus we can identify $\Sigma_{\mathfrak{C}}$ from [HRY17, Definition 2.11] with the full subcategory of $\mathbf{B}_{\mathfrak{C}}$ whose objects have the form $(\{1, \ldots, n\}, \xi)$ for some $n$.

The functor $\Sigma_{\mathfrak{C}} \rightarrow \mathbf{B}_{\mathfrak{C}}$ is, in fact, an equivalence of categories. Everything we're doing in this section could actually be done 'skeletally', that is, by restricting our constructions to $\Sigma_{\mathfrak{C}}$. This would require us to consider graphs with extra structure, namely orderings on each set $\mathrm{nb}(v)$ and on $\precsim(G)$. We've typically taken this approach in earlier work (for example, in [HRY19] which also deals with the undirected context), but will not do so here. This choice allows us to track certain other papers (e.g., Dou17, JK11, Mar16]) more closely.

Notation 2.4. If $Z$ is a subset of $\mathfrak{C}$, we will write $\jmath: Z \hookrightarrow \mathfrak{C}$ for the inclusion.

We now define certain graph groupoids.

Definition 2.5 (Groupoids of colored graphs). Let $\mathfrak{C}$ be a set equipped with an involution $c \mapsto c^{\dagger}$.

- A $\mathfrak{C}$-colored graph is a graph $G$ together with an involutive map $\zeta: A \rightarrow \mathfrak{C}$. 
- Let $\operatorname{gr}_{\mathfrak{C}}$ be the groupoid whose objects are $\mathfrak{C}$-colored graphs and whose isomorphisms $(G, \zeta) \rightarrow\left(G^{\prime}, \zeta^{\prime}\right)$ are graph isomorphisms $z: G \rightarrow G^{\prime}$ so that

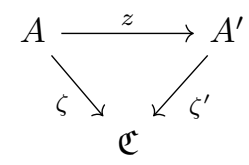

commutes.

- There is a functor, which we call $ð_{\mathfrak{C}}$,

$$
\begin{aligned}
& \operatorname{gr}_{\mathfrak{C}} \stackrel{\partial_{\mathfrak{C}}}{\longrightarrow} \mathbf{B}_{\mathfrak{C}} \\
& (G, \zeta) \longmapsto\left(\delta(G),\left.\zeta\right|_{\partial(G)}\right) .
\end{aligned}
$$

- If $(S, \xi) \in \mathbf{B}_{\mathfrak{C}}$, let $\operatorname{gr}_{\mathfrak{C}}(S, \xi)$ denote the category $(S, \xi) \downarrow \partial_{\mathfrak{C}}$.

Let's unravel this last definition. An object of $\operatorname{gr}_{\mathfrak{C}}(S, \xi)$ consists of a triple $(f, G, \zeta)$ where $(G, \zeta)$ is a $\mathfrak{C}$-colored graph and $f: S \rightarrow \check{\partial}(G)$ is a bijection so that $\left.\zeta\right|_{\delta(G)} \circ f=\xi$. An isomorphism $(f, G, \zeta) \rightarrow\left(f^{\prime}, G^{\prime}, \zeta^{\prime}\right)$ is a graph isomorphism $z: G \rightarrow G^{\prime}$ so that the diagram

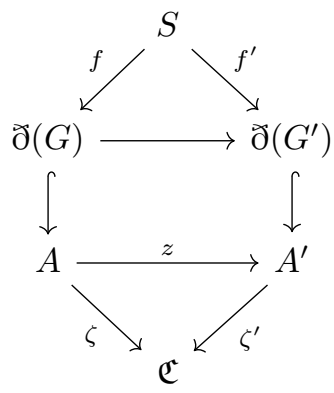

commutes.

Remark 2.6. The groupoid $\operatorname{gr}_{\{*\}}$ is equivalent to $\operatorname{Iso}(\mathbf{U}) \amalg C_{2}$, where $C_{2}$ is the cyclic group of order 2 (considered as a one-object groupoid). Indeed, the only connected graphs that do not already appear in $\mathbf{U}$ are nodeless loops, each of which has a single nontrivial automorphism.

Notice that if $\ell:(S, \xi) \rightarrow\left(S^{\prime}, \xi^{\prime}\right)$ is an isomorphism of $\mathbf{B}_{\mathfrak{C}}$, then we have an induced functor in the reverse direction $\operatorname{gr}_{\mathfrak{C}}(S, \xi) \leftarrow \operatorname{gr}_{\mathfrak{C}}\left(S^{\prime}, \xi^{\prime}\right): \ell^{*}$ taking $(f, G, \zeta)$ to $(f \circ \ell, G, \zeta)$. This is of course an isomorphism, and we write $\ell ! \operatorname{gr}_{\mathfrak{C}}(S, \xi) \rightarrow$ $\operatorname{gr}_{\mathfrak{C}}\left(S^{\prime}, \xi^{\prime}\right)$ for the functor sending $(f, G, \zeta)$ to $\left(f \circ \ell^{-1}, G, \zeta\right)$. In other words, we are considering $\operatorname{gr}_{\mathfrak{C}}(-)$ as a functor from $\mathbf{B}_{\mathfrak{C}}$ to the category of groupoids.

Before approaching the next definition, we introduce some convenient shorthand which we use for the remainder of this subsection. Suppose that $(G, \zeta)$ is a $\mathfrak{C}$-colored graph and $X$ is an object of $\mathbf{E}^{\mathbf{B}_{\mathfrak{c}}}$. We will write $X(v)$ for the object

$$
X(v)=X\left(i \operatorname{nb}(v),\left.\zeta\right|_{i \mathrm{nb}(v)}\right)
$$

in $\mathbf{E}$, suppressing the colored graph $(G, \zeta)$ from the notation. Likewise, for graph groupoids, we write

$$
\operatorname{gr}_{\mathfrak{C}}(v)=\operatorname{gr}_{\mathfrak{C}}\left(i \operatorname{nb}(v),\left.\zeta\right|_{i \operatorname{nb}(v)}\right) .
$$

Definition 2.7 (Decorations). Suppose given an object $X \in \mathbf{E}^{\mathbf{B}_{\mathfrak{C}}}$. 
(1) Let $(G, \zeta)$ be a $\mathfrak{C}$-colored graph. Define the object

$$
X[G, \zeta]=\bigotimes_{v \in V} X(v)=\bigotimes_{v \in V} X\left(i \operatorname{nb}(v),\left.\zeta\right|_{i \mathrm{nb}(v)}\right)
$$

in $\mathbf{E}$.

(2) A decoration of $G$ by $X$ or an $X$-decoration of $G$ consists of an involutive function $\zeta: A(G) \rightarrow \mathfrak{C}$ and an element of $X[G, \zeta]$.

(3) The assignment $(X,(G, \zeta)) \mapsto X[G, \zeta]$ is the object part of a bifunctor

$$
\mathbf{E}^{\mathbf{B}_{\mathfrak{C}}} \times \mathrm{gr}_{\mathfrak{C}} \rightarrow \mathbf{E} .
$$

Construction 2.8 (Colored graph substitution). Suppose that $(G, \zeta)$ is a $\mathfrak{C}$-colored graph. We describe an associated functor

$$
\prod_{v \in V} \operatorname{gr}_{\mathfrak{C}}(v) \rightarrow \operatorname{gr}_{\mathfrak{C}}\left(ð(G),\left.\zeta\right|_{ð(G)}\right) .
$$

Let $\left(m_{v}, H_{v}, \zeta_{v}\right)$ denote an object of $\operatorname{gr}_{\mathfrak{C}}(v)=\operatorname{gr}_{\mathfrak{C}}\left(i \operatorname{nb}(v),\left.\zeta\right|_{i \mathrm{nb}(v)}\right)$, where $m_{v}$ : $i \mathrm{nb}(v) \rightarrow ð\left(H_{v}\right)$ is a bijection satisfying $\left.\zeta\right|_{i \mathrm{nb}(v)}=\zeta_{v} \circ m_{v}$. Then $\prod_{v}\left(m_{v}, H_{v}, \zeta_{v}\right)$

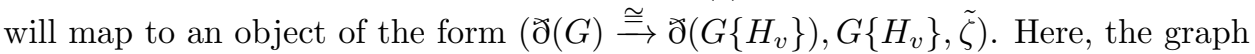
substitution $G\left\{H_{v}\right\}$ is defined using the bijections $m_{v}$. The coloring function $\tilde{\zeta}$ is induced from $\zeta$ and the $\zeta_{v}$. Specifically, the underlying functor part of the graph substitution is described in Definition 1.10 Since colimits in functor category FinSet $^{\mathscr{I}}$ are computed objectwise, we have a coequalizer diagram and an induced map

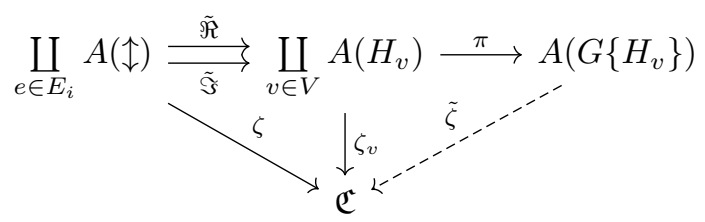

into $\mathfrak{C}$. The map $\partial(G) \rightarrow ð\left(G\left\{H_{v}\right\}\right)$ is the canonical identification of $\precsim(G)$ with ð(G\{H $\left.\left.H_{v}\right\}\right)$.

Graph substitution induces an endofunctor $\top_{\mathfrak{C}}=\top: \mathbf{E}^{\mathbf{B}_{\mathfrak{C}}} \rightarrow \mathbf{E}^{\mathbf{B}_{\mathfrak{C}}}$ where

$$
(\top X)(S, \xi)=\underset{(f, G, \zeta) \in \operatorname{colim}_{\mathfrak{C}}(S, \xi)}{\operatorname{col}} X[G, \zeta] .
$$

Our next goal is to show that $T$ can be given the structure of a monad (Proposition 2.11). Let us first define $\mu: T \top \Rightarrow T$; it is sufficient to define, for $(f, G, \zeta) \in \operatorname{gr}_{\mathfrak{C}}(S, \xi)$ and $X \in \mathbf{E}^{\mathbf{B}_{\mathfrak{C}}}$ the composites

$$
\top X[G, \zeta] \rightarrow \top \top X(S, \xi) \rightarrow \top X(S, \xi) .
$$

We have the following equalities

$$
\begin{aligned}
\top X[G, \zeta]=\bigotimes_{v \in V} \top X(v) & =\bigotimes_{v \in V} \operatorname{colim}_{\substack{\left(m_{v}, H_{v}, \zeta_{v}\right) \in \\
\operatorname{gr}_{\mathfrak{C}}(v)}} X\left[H_{v}, \zeta_{v}\right] \\
& \cong \operatorname{colim}_{\prod_{v} \operatorname{gr}_{\mathfrak{C}}(v)} \bigotimes_{v \in V} X\left[H_{v}, \zeta_{v}\right],
\end{aligned}
$$

where the isomorphism comes from the fact that $\mathbf{E}$ is closed (so $\otimes$ commutes with colimits). Further, we have

$$
\bigotimes_{v \in V(G)} X\left[H_{v}, \zeta_{v}\right] \cong \bigotimes_{w \in V\left(G\left\{H_{v}\right\}\right)} X(w)=X\left[G\left\{H_{v}\right\}, \tilde{\zeta}\right]
$$


where $\tilde{\zeta}$ is the coloring for $G\left\{H_{v}\right\}$ appearing in Construction 2.8. Thus graph substitution provides the first map in the composite

$$
\top X[G, \zeta] \rightarrow \operatorname{gr}_{\operatorname{gr}_{\mathfrak{c}}\left(\widetilde{c}(G),\left.\zeta\right|_{ð(G)}\right)} X\left[K, \zeta^{\prime}\right] \stackrel{\cong}{\rightarrow}(\top X)(S, \xi),
$$

while the second morphism comes from the functor $\operatorname{gr}_{\mathfrak{C}}\left(ð(G),\left.\zeta\right|_{ð(G)}\right) \rightarrow \operatorname{gr}_{\mathfrak{C}}(S, \xi)$ induced by $f:(S, \xi) \rightarrow\left(ð(G),\left.\zeta\right|_{\partial(G)}\right)$.

Remark 2.9. The above degenerates into something much simpler for $(f, G, \zeta) \in$ $\operatorname{gr}_{\mathfrak{C}}(S, \xi)$ when $G$ has no vertices. In that case, both $\top X[G, \zeta]$ and $X[G, \zeta]$ are the tensor unit. Further, what would usually be the structural map $X\left[G\left\{H_{v}\right\}, \tilde{\zeta}\right] \rightarrow$ $(\top X)\left(ð(G),\left.\zeta\right|_{\partial(G)}\right)$ just becomes a map from $X[G, \zeta]$ at the object (id, $\left.G, \zeta\right) \in$ $\operatorname{gr}_{\mathfrak{c}}\left(ð(G),\left.\zeta\right|_{ð(G)}\right)$. That is, 3 factors through this structural map:

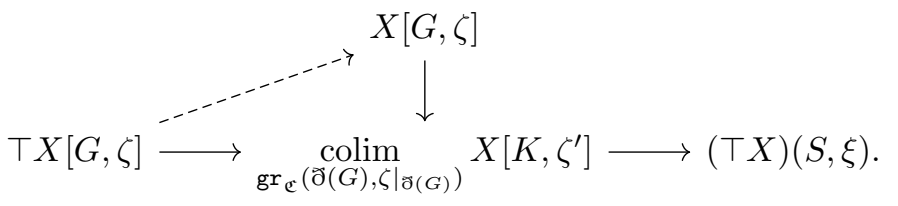

We now turn to the unit $\eta:$ id $\Rightarrow \top$. For this, the following definition is helpful.

Definition 2.10. Let $(S, \xi)$ be an object of $\mathbf{B}_{\mathfrak{C}}$. Recall that the graph $\boldsymbol{w}_{S}$ from Definition 1.3 has a single vertex, $A=2 S$, and $\partial\left(\xi_{S}\right)=S$. There is a unique involutive extension $\xi^{\text {出 }}: 2 S \rightarrow \mathfrak{C}$ of $\xi: S \rightarrow \mathfrak{C}$, namely the one with $\xi^{\text {出 }}(s)=\xi(s)$ and $\xi^{\grave{\omega}}\left(s^{\dagger}\right)=(\xi(s))^{\dagger}$.

If $(S, \xi) \in \mathbf{B}_{\mathfrak{C}}$, then $X\left[\xi_{S}, \xi^{\natural}\right]=X(S, \xi)$. The map

$$
\eta_{(S, \xi)}: X(S, \xi) \rightarrow \top X(S, \xi)
$$

is defined to be the structural map

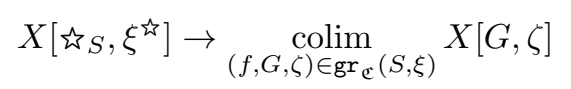

associated with the object $\left(\operatorname{id}_{S}, \boldsymbol{\sim}_{S}, \xi^{\natural}\right) \in \operatorname{gr}_{\mathfrak{C}}(S, \xi)$.

Proposition 2.11. The functor $\top=\top_{\mathfrak{C}}: \mathbf{E}^{\mathbf{B}_{\mathfrak{C}}} \rightarrow \mathbf{E}^{\mathbf{B}_{\mathfrak{C}}}$, coupled with the natural transformations $\mu: \mathrm{T} \top \Rightarrow \mathrm{T}$ and $\eta: \mathrm{id} \Rightarrow \mathrm{T}$, comprise a monad.

Proof. Graph substitution is associative and unital (YJ15, Theorem 5.32; Lemma 5.31]) which implies the result.

Definition 2.12. Given an involutive set of colors $\mathfrak{C}$, the category of algebras over the monad $\left(T_{\mathfrak{C}}, \mu, \eta\right)$ on $\mathbf{E}^{\mathbf{B}_{\mathfrak{C}}}$ is denoted by $\mathbf{M o d O} \mathbf{p}_{\mathfrak{C}}(\mathbf{E})$. Objects of $\mathbf{M o d} \mathbf{O} \mathbf{p}_{\mathfrak{C}}(\mathbf{E})$ are called modular operads in $\mathbf{E}$ with objects $\mathfrak{C}$.

Given a map $f: \mathfrak{C} \rightarrow \mathfrak{D}$ of involutive sets, there is corresponding adjoint pair

$$
f_{!}: \operatorname{ModOp}_{\mathfrak{C}}(\mathbf{E}) \leftrightarrows \operatorname{ModOp}_{\mathfrak{D}}(\mathbf{E}): f^{*}
$$

where $\left(f^{*} X\right)(S, \xi)=X(S, f \circ \xi)$. It is evident that $(g f)^{*}=f^{*} g^{*}$, so it follows that $g ! f_{!}=(g f) !$ 
Definition 2.13. Let $\operatorname{Mod} O \mathbf{p}(\mathbf{E})$ denote the category of all modular operads. If $X$ has objects $\mathfrak{C}$ and $Y$ has objects $\mathfrak{D}$, then

$$
\operatorname{ModOp}(\mathbf{E})(X, Y)=\coprod_{f: \mathfrak{C} \rightarrow \mathfrak{D}} \operatorname{ModOp}_{\mathfrak{C}}(\mathbf{E})\left(X, f^{*} Y\right)
$$

where $f$ ranges over all maps of involutive sets $\mathfrak{C} \rightarrow \mathfrak{D}$. Composition of morphisms is as usual in the Grothendieck construction. More precisely, if iSet is the category of involutive sets, then there is a functor iSet $^{\mathrm{op}} \rightarrow$ Cat that sends $\mathfrak{C}$ to $\operatorname{Mod} \mathbf{O p}_{\mathfrak{C}}(\mathbf{E})$ and $f: \mathfrak{C} \rightarrow \mathfrak{D}$ to $f^{*}$ defined above. Then $\operatorname{ModOp}(\mathbf{E}) \rightarrow$ iSet, sending a modular operad to its involutive set of colors, is the associated Grothendieck (cartesian) fibration.

Each of the categories $\mathbf{M o d O}_{\mathfrak{C}}(\mathbf{E})$ will be complete or cocomplete when $\mathbf{E}$ is. Completeness is standard, while for cocompleteness one should check that $T_{\mathfrak{C}}$ is a finitary monad. In her thesis, Sophie Raynor shows that there is a colored operad whose category of algebras is $\mathbf{M o d} \mathbf{O p}_{\mathfrak{C}}(\mathbf{E})$ Ray18, §4.5], which implies this fact.

Remark 2.14. Since each $f^{*}$ has a left adjoint $f_{!}$, the functor $\operatorname{ModOp}(\mathbf{E}) \rightarrow$ iSet is actually a bifibration (see, for instance, [Jac99, Lemma 9.1.2]). Given any bifibration with bicomplete base and bicomplete fibers, the total category is also bicomplete (this is classical, see Exercise 9.2.4, p.531 of [Jac99]). Since iSet and all $\operatorname{ModO}_{\mathfrak{C}}(\mathbf{E})$ are bicomplete when $\mathbf{E}$ is, it follows that $\operatorname{ModOp}(\mathbf{E})$ is also bicomplete when $\mathbf{E}$ is.

Remark 2.15. The category of colored modular operads of Definition 2.13 was introduced in [JK11, under the name 'compact symmetric multicategories,' using a related monad but only for $\mathbf{E}=$ Set. One benefit to their approach is that it used a single monad, rather than one for each involutive set of colors. One drawback is that it is not clear how to generalize to the cases when $\mathbf{E}$ is different from Set. Note that in the third paragraph of $\S 5$ of [JK11], the monad is not well-defined at level $n=0$; one needs to add in nodeless loops to the collection of graphs to make this correct. An alternative approach can be found in Ray19.

Remark 2.16. At the beginning of the introduction, we said that (monochrome) modular operads may be specified by composition operations and by contraction operations, which satisfy a small collection of axioms. Appropriate presentations appear in the non-skeletal setting in Definition 2.1 of [Dou17] (stable) and Definition A.4 of [Mar16] (unstable). Of course this works just as well for the $\mathfrak{C}$-colored modular operads of Definition 2.12, with the understanding that one should replace finite sets with finite sets over $\mathfrak{C}$ and that compositions and contractions will be defined only when the colorings match; this was laid out in Ray18, §2.2]. All of these references cover the case of non-unital modular operads. To our knowledge there is not a similar presentation for the skeletal context (as in Remark 2.3) in the literature. However, for the case of cyclic operads (with units and involutive color sets), where we have compositions but no contractions, such a system is included in the paper DCH18 of Drummond-Cole and the first author. In any case, we expect that these types of 'biased' definitions of modular operads would play a key role in determining whether modular operads are equivalent to strict inner Kan U-presheaves.

2.2. The modular operad associated to a graph. Let us consider $\mathfrak{C}$-colored modular operads with underlying symmetric monoidal category $\mathbf{E}=$ Set. There is an adjunction

$$
F_{\mathfrak{C}}: \operatorname{Set}^{\mathbf{B}_{\mathfrak{C}}} \rightleftarrows \operatorname{ModO} \mathbf{p}_{\mathfrak{C}}: U_{\mathfrak{C}},
$$


(where $U_{\mathfrak{C}} F_{\mathfrak{C}}=\top_{\mathfrak{C}}$ ) which we can use to produce new modular operads. In particular, if $G$ is a graph then we can produce a modular operad $\langle G\rangle$ whose operations are generated by the vertices of $G$.

Definition 2.17 (The modular operad generated by a graph). Suppose $G$ is a connected ${ }^{4}$ possibly unsafe, graph with set of arcs $A$ and set of vertices $V$.

(1) If $\wp(A)$ is the power set of $A$, we consider the object $\underline{G}_{\wp}$ in $\operatorname{Set}^{\wp(A)}$ satisfying $\underline{G}_{\wp}(Z)$ is a point if $Z=i \mathrm{nb}(v)$ for some $v$, and otherwise $\underline{G}_{\wp}(Z)$ is empty.

(2) The power set $\wp(A)$ of subsets of $A$ includes into the groupoid $\mathbf{B}_{A}$ by sending a subset $Z \subseteq A$ to $(Z, \jmath)$ (see Notation 2.4. We write $\underline{G}_{B} \in \mathbf{S e t}^{\mathbf{B}_{A}}$ for the left Kan extension of $\underline{G}_{\wp}$.

(3) More concretely, $\underline{G}_{B} \in \mathbf{S e t}^{\mathbf{B}_{A}}$ is given by

$$
\underline{G}_{B}(S, \xi)= \begin{cases}\{(v, \xi)\} & \text { if } \xi: S \rightarrow A \text { is injective and } \xi(S)=i \operatorname{nb}(v) \\ \varnothing & \text { otherwise. }\end{cases}
$$

(4) Define a (free $A$-colored) modular operad, the modular operad generated by $G$, as $\langle G\rangle=F_{A}\left(\underline{G}_{B}\right) \in \operatorname{ModOp}_{A}$.

Given that an $\mathfrak{C}$-colored modular operad is an algebra over the monad $\top_{\mathfrak{C}}$ in Section 2.1, we see that an element in $\langle G\rangle$ is represented by a $\underline{G}_{B}$-decorated graph; let us unravel this a bit. If $(S, \xi)$ is an object of $\mathbf{B}_{A}$, then

$$
\langle G\rangle(S, \xi)=\left(\top \underline{G}_{B}\right)(S, \xi)=\underset{(f, K, \zeta) \in \operatorname{corr}_{A}(S, \xi)}{\operatorname{colim}} \underline{G}_{B}[K, \zeta] .
$$

Here, $(K, \zeta)$ is an $A$-colored graph (which may be a nodeless loop, see Example 1.2), $f: S \rightarrow \check{\partial}(K)$ is a bijection so that $\left.\zeta\right|_{\partial(K)} \circ f=\xi$, and

$$
\underline{G}_{B}[K, \zeta]=\prod_{w \in V(K)} \underline{G}_{B}\left(i \operatorname{nb}(w),\left.\zeta\right|_{i \operatorname{nb}(w)}\right) .
$$

Given the structure of $\underline{G}_{B}$, the set $\underline{G}_{B}[K, \zeta]$ will be a point just when, for each $w \in V(K)$, the function $\left.\zeta\right|_{i \mathrm{nb}(w)}$ constitutes a bijection $i \mathrm{nb}(w) \rightarrow i \operatorname{nb}(v)$ for some (unique, since $G$ is connected) vertex $v \in V(G)$. In all other cases, $\underline{G}_{B}[K, \zeta]$ is the empty set.

Remark 2.18. Let $G$ be a safe graph. An important special case of elements of $\langle G\rangle$ come from embeddings in the sense of Definition 1.4. Specifically, if $K$ is an object of $\mathbf{U}$ and $f: K \hookrightarrow G$ is an embedding, then the maps $A(K) \rightarrow A(G)$ and $V(K) \rightarrow V(G)$ constitute a $\underline{G}_{B}$-decoration of $K$. There's a slight ambiguity about where in $\langle G\rangle$ to locate this element, and we make the following choice. We have the factorization

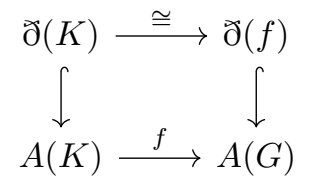

and we write $\left(\left.f\right|_{\partial(K)}\right)^{-1}: \partial(f) \rightarrow ð(K)$ for inverse of the top map. Then we associate to $f$ the object $\left(\left(\left.f\right|_{\partial(K)}\right)^{-1}, K, f\right)$ in $\mathrm{gr}_{A(G)}(ð(f), \jmath)$ (see Notation 2.4 and the discussion following Definition 2.5), so we think of $f$ as representing an

\footnotetext{
${ }^{4}$ This definition is nearly correct for disconnected graphs as well, but does not produce the expected answer when $G$ has more than one isolated vertex.
} 
object of $\langle G\rangle(\partial(f), \jmath)$. There are other choices about where this element should

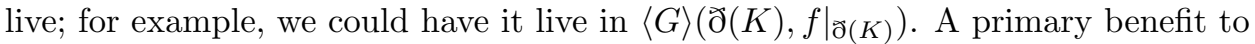
our choice is that it is invariant under isomorphism of embeddings: if $f_{1}: K_{1} \rightarrow G$ and $f_{2}: K_{2} \rightarrow G$ are two embeddings with $f_{1}=f_{2} z$ ( $z$ an isomorphism), then $\partial\left(f_{1}\right)=\partial\left(f_{2}\right)$. The isomorphism $z$ lives in $\mathrm{gr}_{A(G)}\left(\partial\left(f_{1}\right), \jmath\right)$ so $f_{1}$ and $f_{2}$ will represent the same element of $\langle G\rangle\left(\partial\left(f_{1}\right), \jmath\right)$. Had we made the alternative choice, where $f_{j}$ represents an element of $\langle G\rangle\left(\partial\left(K_{j}\right),\left.f_{j}\right|_{\Im\left(K_{j}\right)}\right)$, then these two elements would not even be immediately comparable.

In summary, we've both shown how elements of $\widetilde{\operatorname{Emb}}(G)$ produce elements of $\langle G\rangle$, and also that this association factors through $\operatorname{Emb}(G)$. That is, we have an inclusion

$$
\operatorname{Emb}(G) \subseteq \coprod_{Z \subseteq A}\langle G\rangle(Z, \jmath)
$$

Be careful, though: if $Z \subseteq A$ is of order two, we may have distinct elements of $\langle G\rangle(Z, \jmath)$ which are both represented by embeddings, just as in HRY20, Proposition 1.25].

Example 2.19. Let $G=\uparrow$ be the exceptional edge. We have $A=\{\sharp, b\}$ and $V=\varnothing$. Then $\underline{G}_{B}$ is the initial object in $\mathbf{S e t}^{\mathbf{B}_{A}}$, that is, $\underline{G}_{B}(S, \xi)=\varnothing$ for each finite set $S$ and each function $\xi: S \rightarrow A$. As $F_{A}$ is a left adjoint, this implies that $\langle G\rangle=F_{A}\left(\underline{G}_{B}\right)$ is initial in $\mathbf{M o d O} \mathbf{p}_{A}$. The considerations above show that we have

$$
|\langle G\rangle(S, \xi)|= \begin{cases}1 & \text { if } \xi: S \rightarrow A \text { is bijective } \\ 1 & \text { if } S=\varnothing, \text { and } \\ 0 & \text { otherwise. }\end{cases}
$$

The second line comes from the fact that there are two $A$-colorings of a nodeless loop, but they are isomorphic in $\operatorname{gr}_{A}(\varnothing, \jmath)$. We likewise have two $A$-colorings of the exceptional edge, which are isomorphic in $\mathrm{gr}_{A}$, but are incomparable once we consider the extra structure to make them objects of $\operatorname{gr}_{A}(S, \xi)$ for some $(S, \xi)$. For any $P \in \operatorname{ModO}_{\mathfrak{C}}$, we have $\operatorname{ModO} \mathbf{p}(\langle\uparrow\rangle, P) \cong \mathfrak{C}$ : any map $f$ is determined by $f(\sharp) \in \mathfrak{C}$.

A nodeless loop will also generate the modular operad from this example, as the boundary of $G$ does not factor in the definition of $\langle G\rangle$.

Example 2.20. If $G$ is the isolated vertex ${ }^{0} 0$, then we have $A=\varnothing$ and $V=\{v\}$. The resulting object $\left\langle\xi_{0}\right\rangle$ is in $\mathbf{M o d O} \mathbf{p}_{\varnothing}$, hence only has a single set to define. In this case, $\left\langle\hat{\xi}_{0}\right\rangle\left(\varnothing, \mathrm{id}_{\varnothing}\right)$ is a point. In fact, $\mathbf{M o d O}_{\varnothing}$ is equivalent to the category of sets, and $\left\langle\hat{\tau}_{0}\right\rangle$ is a generator.

We wish to show that the assignment $G \mapsto\langle G\rangle$ is the object part of a functor from $\mathbf{U} \rightarrow$ ModOp. As $\langle G\rangle$ is a free $A$-colored modular operad, it is easy to define maps out of $\langle G\rangle$.

Lemma 2.21. Suppose $G$ is a graph and $P$ is a $\mathfrak{C}$-colored modular operad. A map

$$
f:\langle G\rangle \rightarrow P
$$

is equivalent to the data:

- an involutive function $f_{0}: A \rightarrow \mathfrak{C}$, where $A$ is the set of arcs of $G$, and

- for each vertex $v \in V(G)$, an element $f_{1}(v)$ in $P\left(i \mathrm{nb}(v),\left.f_{0}\right|_{i \mathrm{nb}(v)}\right)$. 


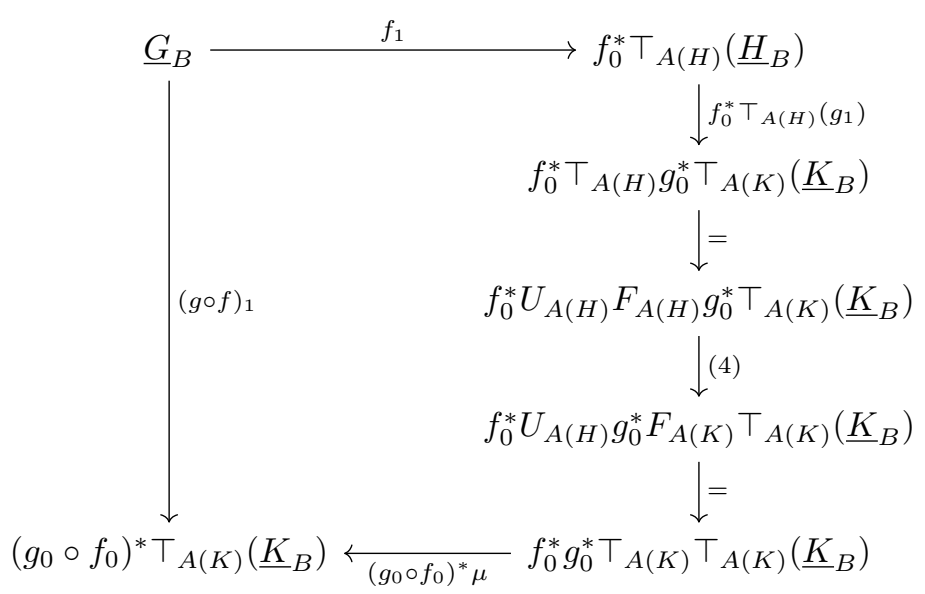

Figure 2. Composition of maps between graphical modular operads

Proof. The first piece of data just comes from the fact that $A$ and $\mathfrak{C}$ are the color sets for these modular operads. The data of a map $\langle G\rangle \rightarrow P$ with underlying color map $f_{0}: A \rightarrow \mathfrak{C}$ is just a map $\langle G\rangle \rightarrow f_{0}^{*} P$ in $\mathbf{M o d O p}_{A}$. But $\langle G\rangle$ is free in $\operatorname{ModO} \mathbf{p}_{A}$, so this just amounts to a map of $\mathbf{B}_{A}$ diagrams $\underline{G}_{B} \rightarrow f_{0}^{*} P$. We of course have

$$
f_{0}^{*} P(S, \xi)=P\left(S, f_{0} \circ \xi\right) .
$$

The result then follows from the description in Definition 2.17 of $\underline{G}_{B}$ as a left Kan extension.

Remark 2.22 (Composition of maps between graphical modular operads). Let us describe composition of maps appearing in Lemma 2.21 whose targets are also modular operads generated by graphs. As might be expected, this looks a bit like Kleisli composition, but adjusted for the fact that ModOp is the Grothendieck construction associated to $\mathfrak{C} \mapsto \mathbf{M o d O}_{\mathfrak{C}}$ (Definition 2.13). Specifically, suppose that $f:\langle G\rangle \rightarrow\langle H\rangle$ and $g:\langle H\rangle \rightarrow\langle K\rangle$ are modular operad maps. By the lemma, this is equivalent to maps

$$
\begin{aligned}
& f_{1}: \underline{G}_{B} \rightarrow f_{0}^{*} \top_{A(H)}\left(\underline{H}_{B}\right) \\
& g_{1}: \underline{H}_{B} \rightarrow g_{0}^{*} \top_{A(K)}\left(\underline{K}_{B}\right)
\end{aligned}
$$

in the diagram categories $\mathbf{S e t}^{\mathbf{B}_{A(G)}}$ and $\mathbf{S e t}^{\mathbf{B}_{A(H)}}$. The map $g_{0}^{*}$ is a functor from Set $^{\mathbf{B}_{A(K)}}$ to $\mathbf{S e t}^{\mathbf{B}_{A(H)}}$ with $\left(g_{0}^{*} X\right)(S, \xi)=X\left(S, g_{0} \circ \xi\right)$; likewise, $g_{0}$ also induces a functor $g_{0}^{*}$ between $\operatorname{ModO}_{A(K)} \rightarrow \mathbf{M o d O p}_{A(H)}$ satisfying $U_{A(H)} g_{0}^{*}=g_{0}^{*} U_{A(K)}$. Applying the first of these to the unit $\eta$ : id $\Rightarrow \top_{A(K)}=U_{A(K)} F_{A(K)}$ for the monad $\top_{A(K)}$ gives a natural transformation

$$
g_{0}^{*} \Rightarrow g_{0}^{*} \top_{A(K)}=g_{0}^{*} U_{A(K)} F_{A(K)}=U_{A(H)} g_{0}^{*} F_{A(K)} .
$$

Taking adjoints gives a natural transformation

$$
F_{A(H)} g_{0}^{*} \Rightarrow g_{0}^{*} F_{A(K)}
$$

of functors $\mathbf{S e t}^{\mathbf{B}_{A(K)}} \rightarrow \operatorname{ModO}_{A(H)}$. To get $(g \circ f)_{1}$, we use the diagram in Figure 2, where $\mu$ is the multiplication of the $\operatorname{monad} \top_{A(K)}$. 
We wish to extend the assignment $G \mapsto\langle G\rangle$ to a functor $\mathbf{U} \rightarrow$ ModOp. As we have seen, defining maps out of $\langle G\rangle$ is straightforward, since $\langle G\rangle$ is free in $\mathbf{M o d} \mathbf{O} \mathbf{p}_{A}$. We use Remark 2.18 to regard embeddings as elements of $\langle G\rangle$.

Definition 2.23 (Assignment on morphisms). Suppose that $\varphi: G \rightarrow G^{\prime}$ is a graphical map in U. Define a morphism of modular operads $f:\langle G\rangle \rightarrow\left\langle G^{\prime}\right\rangle$, using Lemma 2.21, as follows:

- The map of involutive sets $f_{0}: A \rightarrow A^{\prime}$ is just $\varphi_{0}$.

- Each $\varphi_{1}(v) \in \operatorname{Emb}\left(G^{\prime}\right)$ determines an element of $\left\langle G^{\prime}\right\rangle\left(\partial\left(\varphi_{1}(v)\right), \jmath\right)$ by Remark 2.18. There is an isomorphism $\left(ð\left(\varphi_{1}(v)\right), \jmath\right) \cong\left(i \operatorname{nb}(v),\left.\varphi_{0}\right|_{i \operatorname{nb}(v)}\right)$ in $\mathbf{B}_{A^{\prime}}$ by Definition 1.6 (ii), and we let

$$
f_{1}(v) \in\left\langle G^{\prime}\right\rangle\left(i \operatorname{nb}(v),\left.\varphi_{0}\right|_{i \mathrm{nb}(v)}\right) \cong\left\langle G^{\prime}\right\rangle\left(\partial\left(\varphi_{1}(v)\right), \jmath\right)
$$

be the element corresponding to $\varphi_{1}(v) \in \operatorname{Emb}\left(G^{\prime}\right)$.

Each isomorphism of $\mathbf{U}$ maps to an isomorphism of modular operads. In Lemma 2.24 we give a partial converse to this statement. Notice in this lemma that the graphs $G$ and $G^{\prime}$ are in $\mathbf{U}$; in particular, neither of these graphs is a nodeless loop. Of course nodeless loops will generate the same modular operad as the exceptional edge (the initial object in $\mathbf{M o d O}_{2\{*\}}$ as in Example 2.19), though these are not isomorphic graphs. See the paragraph preceding Remark 3.19 where we consider this extension. A discussion on a similar topic in the directed setting appears in Section 2 of [HRY18].

Lemma 2.24. Suppose that $G$ and $G^{\prime}$ are graphs in $\mathbf{U}$. If $f:\langle G\rangle \rightarrow\left\langle G^{\prime}\right\rangle$ is an isomorphism of modular operads, then there exists an isomorphism $\varphi: G \rightarrow G^{\prime}$ in $\mathbf{U}$ so that $\varphi \mapsto f$.

Proof. As we know $f_{0}: A \rightarrow A^{\prime}$ is an involutive isomorphism, we replace (strictly for convenience) $G^{\prime}$ with an isomorphic graph $H$ which has the same set of arcs as $G$ and the same vertices as $G^{\prime}$.

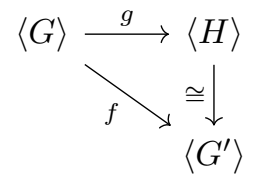

It is sufficient to show that the induced isomorphism $g:\langle G\rangle \rightarrow\langle H\rangle$ comes from an isomorphism in $\mathbf{U}$. Let $h$ be the inverse to $g$.

We are now just working in $\mathbf{M o d O p}_{A}$, the category of algebras for $\top_{A}=\top$. The composition diagram in Remark 2.22 simplifies to the usual Kleisli composition diagrams.
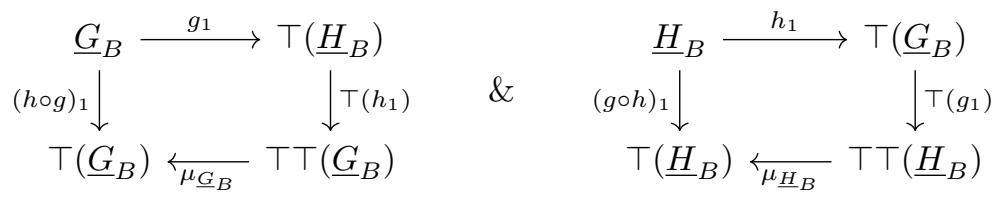

By the assumption that $h=g^{-1}$, we have that $(h \circ g)_{1}=\eta_{\underline{G}_{B}}$ and $(g \circ h)_{1}=\eta_{\underline{H}_{B}}$, where $\eta$ is the unit of the monad.

Suppose that $v$ is a vertex of $G$; then the map

$$
g_{1}: \underline{G}_{B}(i \operatorname{nb}(v), \jmath) \rightarrow \top\left(\underline{H}_{B}\right)(i \operatorname{nb}(v), \jmath)
$$




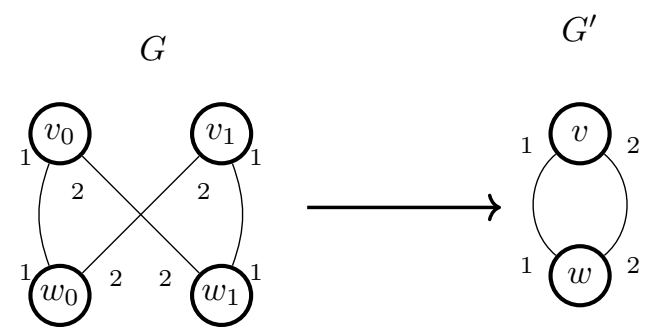

Figure 3. An étale map that is not an embedding.

takes $(v, \jmath)$ (see Definition 2.17) to some $\underline{H}_{B}$-decorated graph $K$. The (connected) graph $K$ must have at least one vertex, since $(h \circ g)_{1}(v, \jmath)$ is a star, thus is not an edge. Similarly, in the right-hand diagram we have that $h_{1}$ only produces graphs that have at least one vertex.

Now if $g_{1}(v, \jmath)$ has more than one vertex, or a loop at a vertex, then so does $(h \circ g)_{1}(v, \jmath)$ since $h_{1}$ does not send vertices to edges and thus these are preserved by $\mu$. Since $(h \circ g)_{1}(v, \jmath)$ is $\left(\boldsymbol{w}_{i \mathrm{nb}(v)}, \xi^{\text {访}}\right)$, we thus know that $g_{1}(v, \jmath)$ is a $\underline{H}_{B}$-decorated star. Likewise, $h_{1}$ produces only $\underline{G}_{B}$-decorated stars.

It follows that $g_{1}$ and $h_{1}$ induce a bijection between $V(G)$ and $V(H)$. If $w$ is the vertex of $H$ that is associated to $g_{1}(v)$, then we have $i: \operatorname{nb}(w) \rightarrow i \mathrm{nb}(v)$ is a bijection, so $\operatorname{nb}(v)=\operatorname{nb}(w)$. Thus $g$ induces an isomorphism $G \cong H$ of graphs.

Proposition 2.25. The assignment $G \mapsto\langle G\rangle$ on objects from Definition 2.17 and the assignment on graphical maps from Definition 2.23 constitute a faithful functor $J: \mathbf{U} \rightarrow \mathbf{M o d O p}$ which is injective on isomorphism classes of objects.

Proof. The fact that $\mathbf{U} \rightarrow$ ModOp is a functor follows by comparing Figure 2 from Remark 2.22 with the composition for $\mathbf{U}$ (Definition 1.7). Lemma 2.24 shows that the functor is injective on isomorphism classes of objects. To see that the functor is faithful, suppose that $\varphi$ and $\psi$ are elements of $\mathbf{U}\left(G, G^{\prime}\right)$ which map to the same morphism $f:\langle G\rangle \rightarrow\left\langle G^{\prime}\right\rangle$. Then the maps on color sets $\varphi_{0}$ and $\psi_{0}$ are equal. Further, for each $v \in V(G)$ the element $f_{1}(v)$ in

$$
\operatorname{Emb}\left(G^{\prime}\right) \subseteq \coprod_{Z \subseteq A^{\prime}}\left\langle G^{\prime}\right\rangle(Z, \jmath)
$$

is equal to both $\varphi_{1}(v)$ and $\psi_{1}(v)$.

Example 2.26. The functor $J: \mathbf{U} \rightarrow$ ModOp is not full. Here we give two examples.

- Consider the two graphs from Figure 3. There is a map from $\langle G\rangle$ to $\left\langle G^{\prime}\right\rangle$ sending generators to generators, where each $v_{j}$ goes to $v$ and each $w_{j}$ goes to $w$, but there is no graphical map $G \rightarrow G^{\prime}$ which has this behavior. This example was explained to us by J. Kock, as an illustration of the difference between étale and embedding.

- There is a map $\left\langle\xi_{0}\right\rangle \rightarrow\langle\hat{\rangle}\rangle$ which takes the unique vertex of $\xi_{0}$ (see Example 2.20 to the unique element in $\langle\downarrow\rangle(\varnothing, \jmath)$ (see Example 2.19). In contrast, there are no maps $\boldsymbol{i}_{0} \rightarrow \uparrow$ in $\mathbf{U}$. 


\section{THE NERVE THEOREM}

At this point, we have defined a functor $J: \mathbf{U} \rightarrow$ ModOp. One can consider the associated singular functor, or nerve functor, which is specified by $N(-)=\operatorname{hom}(J,-)$ and goes from ModOp to the category of $\mathbf{U}$-presheaves. The aim of this section is to prove Theorem 3.6 , which says both that $N$ is fully-faithful and identifies the essential image.

Definition 3.1. The nerve functor for modular operads is the functor

$$
\operatorname{ModOp} \stackrel{N}{\longrightarrow} \widehat{\mathbf{U}}
$$

which is given on a modular operad $P$ and a graph $G \in \mathbf{U}$ by

$$
N P_{G}=\operatorname{ModOp}(\langle G\rangle, P) \text {. }
$$

Here $\langle G\rangle$ is the modular operad generated by $G \in \mathbf{U}$ (Definition 2.17).

An element of the set $N P_{G}$ is a $P$-decoration of the graph $G$ (Definition 2.7). This description comes directly from the fact that $N P_{G}=\operatorname{ModOp}(\langle G\rangle, P)$ and the description of a graphical map given in Lemma 2.21.

Remark 3.2. Given a graph $G \in \mathbf{U}$, we now have two ways to assign an object of $\widehat{\mathbf{U}}$ to $G$. The first is to consider the representable presheaf $\mathrm{U}[G]$, while the second is to first consider the modular operad $\langle G\rangle$ and then take the nerve. In light of Example 2.26, we do not expect them to always be the same. The representable $\mathrm{U}[G]$ is always a subobject of $N\langle G\rangle$ (since $J$ is faithful), but, in fact, they nearly never coincide. To see this, let $K$ be the loop with one vertex and let $k$ be one of the two arcs of $K$. By Lemma 2.21 for each arc $a$ of $G$ there is map $f:\langle K\rangle \rightarrow\langle G\rangle$ which sends $k$ to $a$ and the unique vertex of $K$ to the edge spanned by $a$. This type of collapse behavior is precisely what is prohibited by (iii) of Definition 1.6. Thus the inclusion $\mathrm{U}[G]_{K} \subseteq N\langle G\rangle_{K}$ is strict as long as the arc set of $G$ is non-empty. On the other hand, we have $\mathrm{U}\left[\boldsymbol{i}_{0}\right]=N\left\langle\boldsymbol{\tau}_{0}\right\rangle$.

Remark 3.3. Suppose we are given an object $(S, \xi)$ of $\mathbf{B}_{\mathfrak{C}}$, and let $\boldsymbol{w}_{S}$ be the graph from Definition 1.3 Recall from Example 1.2 that we write $A(\uparrow)=\{\sharp, b\}$ and define, for each $s \in S$, an embedding $h_{s}: \uparrow \rightarrow\{S$ which sends $\sharp$ to $s$. There is a natural map

$$
\ell_{S}: N P_{\text {々 } S} \rightarrow\left(N P_{\uparrow}\right)^{S}
$$

which takes an element $x$ to the function $\left(s \mapsto x \circ h_{s}\right) \in \operatorname{hom}\left(S, N P_{\uparrow}\right)$. Under the identifications $N P_{\uparrow}=\operatorname{ModOp}(\langle\uparrow\rangle, P)=\operatorname{hom}(\{\sharp\}, \mathfrak{C})=\mathfrak{C}$, we may regard the function $\xi: S \rightarrow \mathfrak{C}$ as an element of the codomain of $\ell_{S}$. The preimage of $\xi$ under $\ell_{S}$ is precisely $P(S, \xi)$. That is, $P(S, \xi)$ is part of the following pullback diagram.

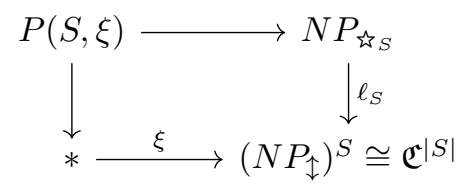

We will use this frequently in what follows.

The Segal core inclusions Sc $[G] \hookrightarrow \mathrm{U}[G]$, from Definition 1.10 , are induced by the embeddings $\iota_{v}: i_{v} \rightarrow G$ (Definition 1.3). This allows us to give the following generalization of the classical Segal condition for categories. 
Definition 3.4 (Segal objects). Suppose that $X$ is a U-presheaf (in Set).

- The Segal map at $G$ is the map

$$
\widehat{\mathbf{U}}(\mathrm{U}[G], X) \longrightarrow \widehat{\mathbf{U}}(\mathrm{Sc}[G], X)
$$

induced by the Segal core inclusion $\mathrm{Sc}[G] \hookrightarrow \mathrm{U}[G]$.

- The presheaf $X$ is said to satisfy the Segal condition if, for every $G \in \mathbf{U}$, the Segal map at $G$ is a bijection.

The reader familiar with the work of Chu and Haugseng may wonder about the relation of this definition with [CH19, Definition 2.6]. As observed in Example 3.12 of [CH19, the category $\mathbf{U}^{\text {op }}$ admits the structure of an 'algebraic pattern.' A presheaf $X$ satisfies the Segal condition (in our sense) if and only if it is a ' $\mathbf{U}^{\text {op }}$-Segal object in Set.'

Remark 3.5. If $X$ is an object of $\widehat{\mathbf{U}}$ and $G$ is $\hat{\Sigma}_{n}$ or $\uparrow$, then the Segal map at $G$ is a bijection.

We are now prepared to state the first main theorem of this paper.

Theorem 3.6. The nerve functor $N: \mathbf{M o d O p} \rightarrow \widehat{\mathbf{U}}$ is fully faithful. Moreover, the following statements are equivalent for $X \in \widehat{\mathbf{U}}$.

(1) There exists a modular operad $P$ and an isomorphism $X \cong N P$.

(2) $X$ satisfies the Segal condition.

We will need a bit of scaffolding before we can approach the proof of this theorem, which appears below.

Suppose that $G$ is a graph with at least one vertex. As in Definition 1.10 , for each internal edge $e \in E_{i}$, we choose an ordering $e=\left[x_{e}^{1}, x_{e}^{2}\right]$ for the two-element equivalence class of arcs comprising $e$.

- Write $\Re_{e}: \uparrow \rightarrow \xi_{t x_{e}^{1}}$ for the embedding that sends $\sharp$ to $\left(x_{e}^{1}\right)^{\dagger} \in ð\left(\boldsymbol{\mho} t x_{e}^{1}\right)$.

- Write $\Im_{e}: \uparrow \rightarrow{ }^{2} t x_{e}^{2}$ for the embedding that sends $\sharp$ to $x_{e}^{2} \in D\left(\boldsymbol{w}^{2} t x_{e}^{2}\right)$.

Lemma 3.7. Suppose that $P$ is a modular operad and $G$ is a graph with at least one vertex. There is an equalizer diagram

$$
N P_{G} \longrightarrow \prod_{v \in V} N P_{\mho_{v}} \stackrel{\Re^{*}}{\underset{\Im^{*}}{\longrightarrow}} \prod_{e \in E_{i}} N P_{\uparrow}
$$

where $\Re^{*}$ and $\Im^{*}$ are defined so that the diagrams

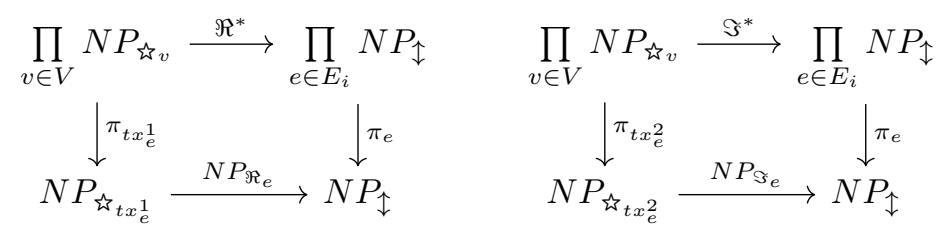

commute for each $e \in E_{i}$.

Proof. Combine Remark 3.3 with Lemma 2.21 .

Lemma 3.8. The nerve of a modular operad satisfies the Segal condition. 
Proof. If $P$ is a modular operad, then

$$
\widehat{\mathbf{U}}(\mathrm{Sc}[G], N P)=\widehat{\mathbf{U}}(\operatorname{coeq}(\Re, \Im), N P) \cong \operatorname{eq}(\widehat{\mathbf{U}}(\Re, N P), \widehat{\mathbf{U}}(\Im, N P)) .
$$

Here $\widehat{\mathbf{U}}(\Re, N P)$ is the top map of the following commutative diagram

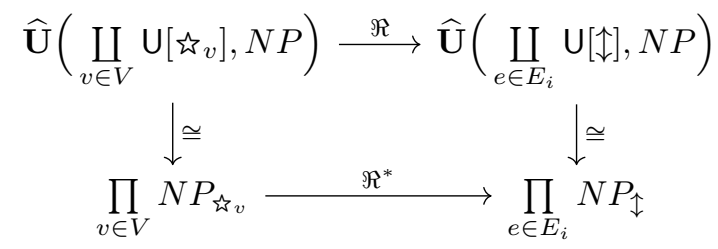

and likewise for $\widehat{\mathbf{U}}(\Im, N P)$. By Lemma 3.7, the equalizer in $(6)$ coincides with $N P_{G}$.

Let us now verify the first statement in Theorem 3.6

Proposition 3.9. The nerve functor $N: \operatorname{ModOp} \rightarrow \widehat{\mathbf{U}}$ is fully faithful.

Proof. Throughout, let $P$ be in $\operatorname{ModO}_{\mathfrak{C}}$ and $Q$ be in $\mathbf{M o d O}_{\mathfrak{D}}$. First we will show that the nerve functor is faithful. Suppose we are given $f, g: P \rightarrow Q$ in ModOp with the property that $N f=N g$. In particular, $f$ and $g$ are equal as involutive functions from $N P_{\uparrow}=\mathfrak{C}$ to $N Q_{\uparrow}=\mathfrak{D}$. As we mentioned in Remark 3.3 . the set $P(S, \xi)$ is the pullback of

$$
N P_{\text {々 } S} \longrightarrow\left(N P_{\uparrow}\right)^{S} \stackrel{\xi}{\longleftarrow} *
$$

and similarly for $Q(S, f \circ \xi)=Q(S, g \circ \xi)$. As we have a commutative diagram

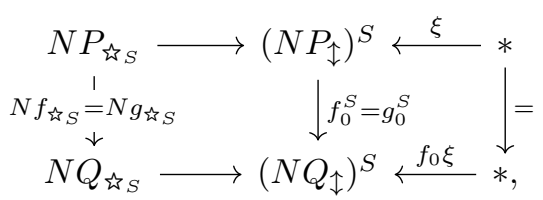

it follows that $f=g$ on each set $P(S, \xi)$. Thus $f$ and $g$ are identical.

To show that the nerve functor is full, now suppose we have a map $\tilde{f}: N P \rightarrow N Q$ in $\widehat{\mathbf{U}}$. We wish to exhibit a modular operad map $f: P \rightarrow Q$ so that $\tilde{f}=N f$. By definition, the map $\tilde{f}: N P_{\mathfrak{1}} \rightarrow N Q_{\uparrow}$ is a map of involutive sets $f_{0}: \mathfrak{C} \rightarrow \mathfrak{D}$.

Similar to previous argument, we know that for each $m$ we have a map of diagrams

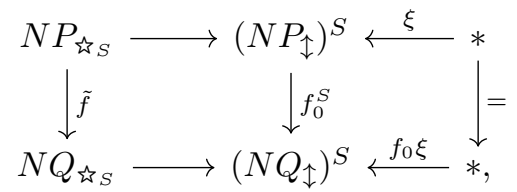

which induces a map of pullbacks $f: P(S, \xi) \rightarrow Q\left(S, f_{0} \xi\right)$.

We've now defined a map $f$ in $\mathbf{E}^{\mathbf{B}_{\mathfrak{C}}}$ from $P$ to $f_{0}^{*} Q$. It remains to show that $f$ is modular operad map, at which point it is automatic that $N f=\tilde{f}$. This amounts to 
showing that the diagram

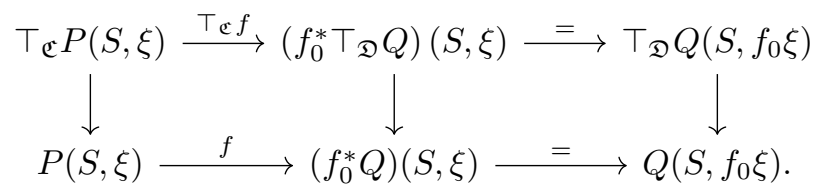

commutes, where the vertical maps $\top_{\mathfrak{C}} P \rightarrow P$ and $\top_{\mathfrak{D}} Q \rightarrow Q$ are the algebra structure maps for $P$ and $Q$.

Consider an object $(h, G, \zeta) \in \mathrm{gr}_{\mathfrak{c}}(S, \xi)$; that is, $(G, \zeta)$ is a $\mathfrak{C}$-colored graph and $h: S \rightarrow ð(G)$ is a bijection so that $\left.\zeta\right|_{\partial(G)} \circ h=\xi$. It suffices, by Definition 2.7 to show that for any such object $(h, G, \zeta)$ that the diagram

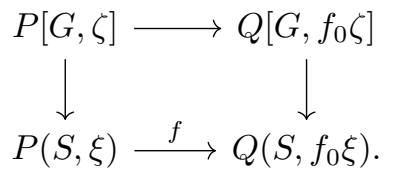

commutes. This is a automatic, as this is a sub-diagram of

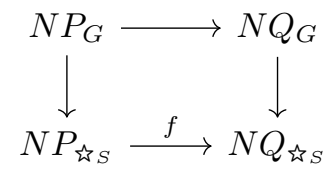

where is $S \rightarrow G$ is the active map determined by $h: S \rightarrow \partial(G)$ (Definition 1.9).

3.1. The modular operad associated to a Segal presheaf. As we saw in Lemma 3.8, the nerve functor factors through the full subcategory of Segal presheaves. We now turn to the last remaining part of Theorem 3.6, namely that every Segal presheaf is (up to isomorphism) the nerve of a modular operad. This requires a construction $X \rightsquigarrow M_{X}$ taking a Segal presheaf to a modular operad.

It is technically convenient to work with the extended graphical category $\widetilde{\mathbf{U}}$ in this section. In a moment, we will fix a Segal $\widetilde{\mathbf{U}}$-presheaf $X$ and endeavor to define $M=M_{X}$, which we call the modular operad associated to $X$ (Definition 3.17). As the underlying object of $M$ is defined via certain pullbacks (Definition 3.12, our construction will only produce an isomorphism class, and is invariant under isomorphism of $\widetilde{\mathbf{U}}$-presheaves. Thus the following remark is harmless.

Remark 3.10. If $Y$ is a Segal $\mathbf{U}$-presheaf, then its right Kan extension $\iota_{*} Y$ along the inclusion $\iota: \mathbf{U} \rightarrow \widetilde{\mathbf{U}}$ is a Segal $\widetilde{\mathbf{U}}$-presheaf [HRY20, Theorem 4.12]. By definition, the modular operad associated to $Y$ is just the modular operad associated to the Segal $\widetilde{\mathbf{U}}$-presheaf $\iota_{*} Y$ (Definition 3.17). On the other hand, if $X$ is a Segal $\widetilde{\mathbf{U}}$-presheaf, then its restriction $\iota^{*} X$ is a Segal $\mathbf{U}$-presheaf and $X \cong \iota_{*} \iota^{*} X$. Thus the modular operad associated to $X$ is the the same as the modular operad associated to the restriction $\iota^{*} X$.

Notation 3.11. If $G$ is a graph in $\widetilde{\mathbf{U}}$, then we will write $\operatorname{Sc}[G] \subseteq \widetilde{\mathrm{U}}[G]$ for the relevant subobject of the representable object. When $G$ a safe graph, this is just the left Kan extension of the usual inclusion Sc $[G] \rightarrow \mathrm{U}[G]$ (Definition 1.10 ), while if $G$ is a nodeless loop then it is of the form $\widetilde{\mathrm{U}}[\uparrow] \rightarrow \widetilde{\mathrm{U}}[G]$. If $X$ is a $\widetilde{\mathbf{U}}$-presheaf, we write

$$
X_{\mathrm{Sc}[G]}=\operatorname{hom}(\mathrm{Sc}[G], X) \text {. }
$$


Fix an arbitrary $\widetilde{\mathbf{U}}$-presheaf $X$ satisfying the Segal condition, and let $\mathfrak{C}$ be the involutive set $X_{\uparrow}$. We start by constructing the underlying object $M$ in $\mathbf{S e t}^{\mathbf{B}_{\mathfrak{c}}}$.

Definition 3.12. For each function $\xi$ from a set $S$ to $X_{\downarrow}=\mathfrak{C}$ we define a set $M(S, \xi)$ as the pullback of

$$
X_{\text {出 }} \longrightarrow\left(X_{\uparrow}\right)^{S} \stackrel{\xi}{\longleftarrow} * .
$$

This defines an object $M$ in $\mathbf{S e t}^{\mathbf{B}_{\mathfrak{C}}}$.

In particular, $M(\varnothing, \jmath)$ is isomorphic to $X_{\text {织 }}$.

In order to now exhibit the object $M$ as an $\top_{\mathfrak{C}}$-algebra, we need to produce a map $\gamma: \top M \rightarrow M$. We again follow the notation that was introduced just before Definition 2.7 and abbreviate, for a $\mathfrak{C}$-colored graph $(G, \zeta)$

$$
X(v)=X\left(i \operatorname{nb}(v),\left.\zeta\right|_{i \mathrm{nb}(v)}\right) \quad \& \quad \operatorname{gr}_{\mathfrak{C}}(v)=\operatorname{gr}_{\mathfrak{C}}\left(i \operatorname{nb}(v),\left.\zeta\right|_{i \operatorname{nb}(v)}\right) .
$$

Let $(f, G, \zeta)$ be an object of $\operatorname{gr}_{\mathfrak{C}}(S, \xi)$ (that is, $(G, \zeta)$ is a $\mathfrak{C}$-colored graph and $f: S \rightarrow \partial(G)$ is a bijection with $\left.\left.\zeta\right|_{\partial(G)} \circ f=\xi\right)$. Since $X$ satisfies the Segal condition and $M(v)$ is a subset of $X_{\text {出 } v}$, we have an inclusion

$$
M[G, \zeta]=\prod_{v \in V} M(v) \hookrightarrow X_{G} .
$$

Note that when $G$ has an empty vertex set, then $M[G, \zeta]$ is a one-point set and this inclusion is essentially equivalent to the coloring $\zeta$.

Definition 3.13 (Action on $M$ ). We define the algebra structure map $\gamma: \top M \rightarrow M$.

- Suppose that $(f, G, \zeta)$ is an object of $\operatorname{gr}_{\mathfrak{C}}(S, \xi)$. We have the following commutative diagram

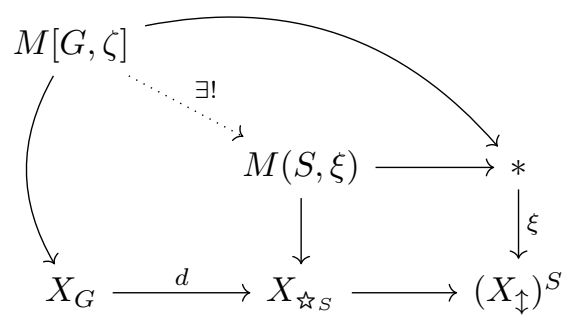

where the bottom square is the pullback used to define $M(S, \xi)$ and the map $d: X_{G} \rightarrow X_{\text {« } S}$ is induced by the active map is $\rightarrow G$ coming from the bijection $f: S \rightarrow \check{\partial}(G)$. We write $\gamma_{f, G, \zeta}$ for the induced map $M[G, \zeta] \rightarrow M(S, \xi)$.

- Since $\top M(S, \xi)=\operatorname{colim}_{\mathrm{gr}_{\mathfrak{c}}(S, \xi)} M[G, \zeta]$ we have defined a map $\top M(S, \xi) \rightarrow$ $M(S, \xi)$ on each component of the colimit and this can be extended to the whole colimit. Since $(S, \xi)$ was arbitrary, we have a map

$$
\gamma: \top M \rightarrow M
$$

in $\mathbf{B}_{\mathfrak{C}}$.

In other words, the structure map $\gamma$ is ultimately induced by the composites (see Notation 3.11

$$
M[G, \zeta] \subseteq X_{\mathrm{Sc}[G]} \stackrel{\cong}{\cong} X_{G} \longrightarrow X_{\text {گ } G}
$$


where $\boldsymbol{\zeta}_{G}=\boldsymbol{\zeta}_{\partial(G)} \rightarrow G$ is the active map induced by the identity on $\partial(G)$.

It remains to show that the map $\gamma$ from Definition 3.13 turns $M$ into a $T$-algebra. Let us first address the unit axiom.

Lemma 3.14. The diagram

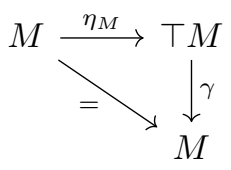

commutes.

Proof. The composite is

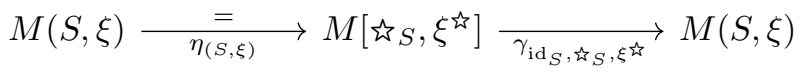

Of course when $G=\xi_{S}$ and $d$ is the identity in Definition 3.13 , then the identity map makes the diagram commute (hence is the unique map making the diagram commute). It follows that the composite (8) is the identity on $M(S, \xi)$.

It remains to show that the diagram

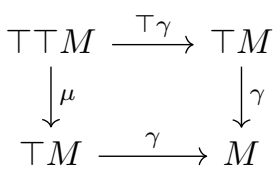

commutes, where $\gamma: \top M \rightarrow M$ is the proposed $\top$-algebra structure map from Definition 3.13. It is enough to show, for each $\mathfrak{C}$-colored graph $(G, \zeta)$, that the diagram commutes when restricted to $(\top M)[G, \zeta] \rightarrow(\top \top M)(ð(G), \jmath)$ coming from considering $\left(\operatorname{id}_{\check{\partial}(G)}, G, \zeta\right)$ as an object of $\operatorname{gr}_{\mathfrak{C}}(\check{\partial}(G), \jmath)$.

Lemma 3.15. If $G$ has no vertices (that is, if $G$ is a nodeless loop or the exceptional edge), then the diagram

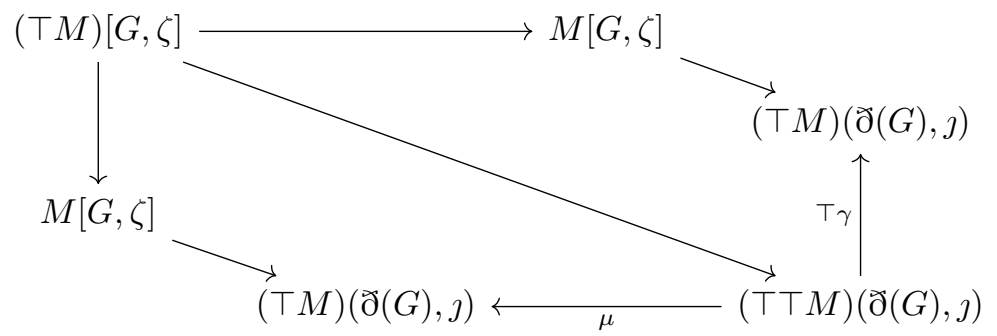

commutes. Here, the diagonal maps are the structural maps for the colimits and the top and left maps are just the unique map on the point. This implies that (9) commutes when restricted to $(\top M)[G, \zeta]$.

Proof. The long diagonal followed by either $\top \gamma$ or $\mu$ factors through the structural map

$$
M[G, \zeta] \rightarrow \underset{\left(f, K, \zeta^{\prime}\right) \in \operatorname{gr}_{\mathfrak{C}}(ð(G), \jmath)}{\operatorname{colim}} M\left[K, \zeta^{\prime}\right]=(\top M)(\check{\partial}(G), \jmath)
$$

at $(\mathrm{id}, G, \zeta) \in \operatorname{gr}_{\mathfrak{C}}(\partial(G), \jmath)$. For $\top \gamma$ this follows because $\top \gamma$ is is given componentwise on

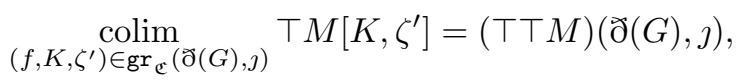


while for $\mu$ it follows from Remark 2.9 . But $M[G, \zeta]$ is a point, so there is exactly one way for a function for factor through this structural map.

Proposition 3.16. The pair $(M, \gamma)$ (from Definition 3.12 and Definition 3.13) is an algebra over $\top_{\mathfrak{C}}$.

Definition 3.17. The pair $(M, \gamma)$ is called the modular operad associated to $X$.

Proof of Proposition 3.16. By Lemma 3.14 we know that this pair satisfies the unit axiom. Thus we must show that $(9)$ commutes, and it is enough to show that it commutes when restricted along the structural maps $(\top M)[G, \zeta] \rightarrow(\top \top M)(\partial(G), \jmath)$. Lemma 3.15 covers the case when $G$ has no vertices, so from now on we assume that $G$ has at least one vertex. In particular, $G$ is an object of $\mathbf{U}$.

The object $(\top M)[G, \zeta]$ is (using the shorthand from (7) appearing before Definition 3.13

$$
\prod_{v \in G} \top M(v)=\prod_{v \in G} \operatorname{colim}_{\substack{\left(m_{v}, H_{v}, \zeta_{v}\right) \in \\ \operatorname{gr}_{\mathfrak{C}}(v)}} M\left[H_{v}, \zeta_{v}\right] ;
$$

we fix a collection $\left(m_{v}, H_{v}, \zeta_{v}\right) \in \operatorname{gr}_{\mathfrak{C}}(v)$ and show that $(9)$ commutes when restricted to the natural map

$$
\prod_{v \in G} M\left[H_{v}, \zeta_{v}\right] \rightarrow(\top M)[G, \zeta] \rightarrow(\top \top M)(ð(G), \jmath) .
$$

Composing with the arrow on the left of $(9)$ factors as

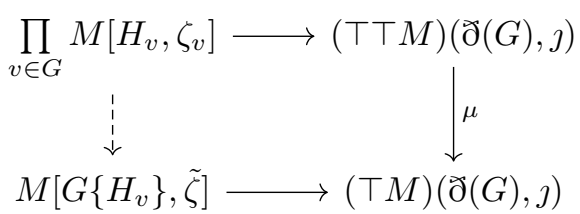

where $\tilde{\zeta}$ is from Construction 2.8 The dashed map comes as follows: each $M\left[H_{v}, \zeta_{v}\right]$ is a subset of

$$
X_{\mathrm{Sc}\left[H_{v}\right]}=\operatorname{hom}\left(\mathrm{Sc}\left[H_{v}\right], X\right) \stackrel{\cong}{\leftrightarrows} X_{H_{v}}
$$

while $M\left[G\left\{H_{v}\right\}, \tilde{\zeta}\right]$ is a subset of

$$
X_{\mathrm{Sc}\left[G\left\{H_{v}\right\}\right]} \cong X_{G\left\{H_{v}\right\}} .
$$

We have a coequalizer diagram

$$
\coprod_{e \in E_{i}} \widetilde{\mathrm{U}}[\uparrow] \stackrel{\Re}{\underset{\Im}{\longrightarrow}} \coprod_{v \in V} \mathrm{Sc}\left[H_{v}\right] \longrightarrow \mathrm{Sc}\left[G\left\{H_{v}\right\}\right],
$$

and by applying hom $(-, X)$, we have a monomorphism $X_{\mathrm{Sc}\left[G\left\{H_{v}\right\}\right]} \hookrightarrow \prod_{v \in G} X_{\mathrm{Sc}\left[H_{v}\right]}$. Compatibility at the boundaries of the $H_{v}$ implies that the images of the monomorphisms

$$
M\left[G\left\{H_{v}\right\}, \tilde{\zeta}\right] \hookrightarrow X_{\mathrm{Sc}\left[G\left\{H_{v}\right\}\right]} \hookrightarrow \prod_{v \in G} X_{\mathrm{Sc}\left[H_{v}\right]}
$$

and $\prod M\left[H_{v}, \zeta_{v}\right] \hookrightarrow \prod X_{\mathrm{Sc}\left[H_{v}\right]}$ coincide. This provides the dashed map in (10).

The left bottom composite of $(9)$ is induced from the zigzag

$$
\coprod_{v \in G} \mathrm{Sc}\left[H_{v}\right] \rightarrow \mathrm{Sc}\left[G\left\{H_{v}\right\}\right] \rightarrow \widetilde{\mathrm{U}}\left[G\left\{H_{v}\right\}\right] \leftarrow \widetilde{\mathrm{U}}\left[\tilde{z}_{G}\right]
$$


(where $\varsigma_{G} \rightarrow G\left\{H_{v}\right\}$ is the active map arising from $\precsim(G) \cong \check{\partial}\left(G\left\{H_{v}\right\}\right)$ ), that is, this zigzag induces

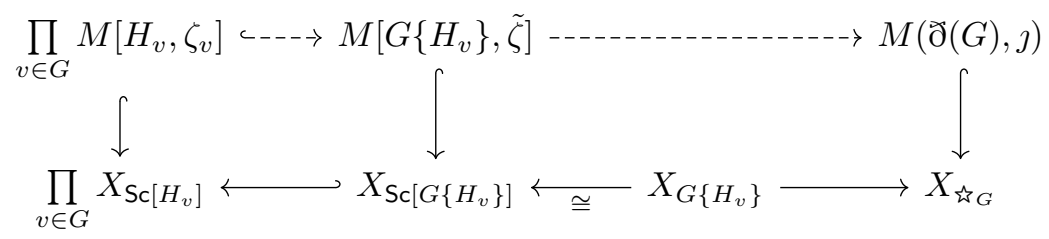

Let us turn to the top right of $(9)$. The top arrow arises from the diagram

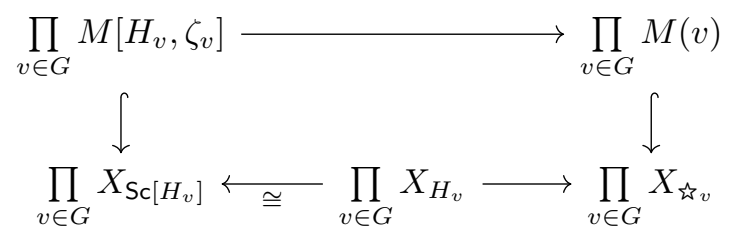

where $\tau_{v} \rightarrow H_{v}$ is the active map determined by $\partial\left(\iota_{v}\right)=\operatorname{nb}(v)^{\dagger} \cong i \operatorname{nb}(v) \stackrel{m_{v}}{\longrightarrow}$ $\precsim\left(H_{v}\right)$ for each $v$. The vertical inclusion on the right factors through $X_{\mathrm{Sc}[G]}$. The arrow on the right of (9) then comes from the diagram

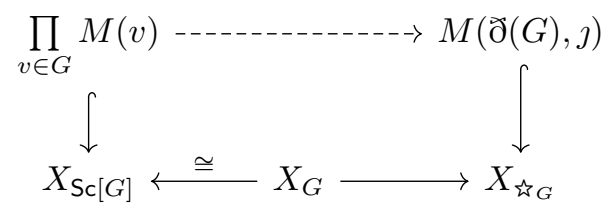

We thus deduce commutativity of $(9)$ from commutativity of the following diagram of $\widetilde{\mathbf{U}}$-presheaves

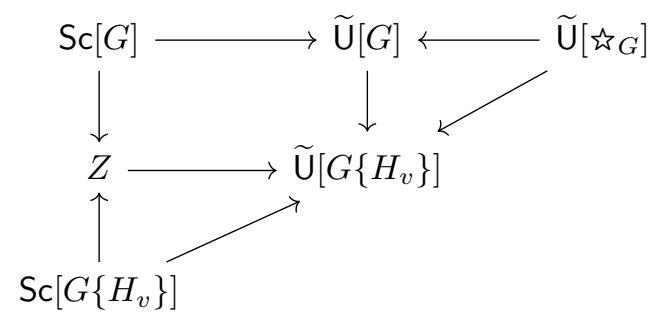

where the zig-zag on the left comes from the following pair of maps of coequalizers

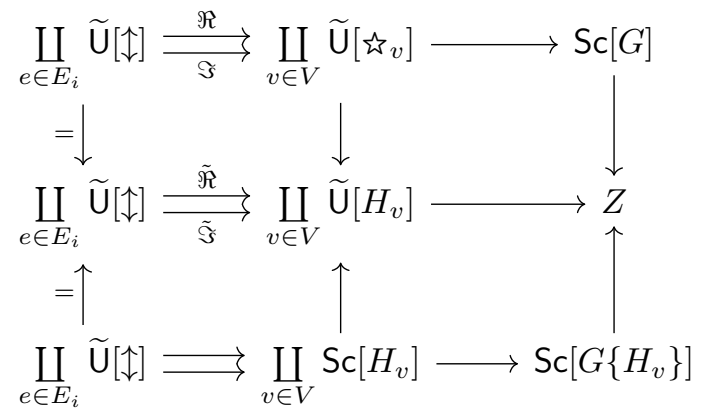


But commutativity of (11) is relatively straightforward. For instance, commutativity of the square follows from commutativity of

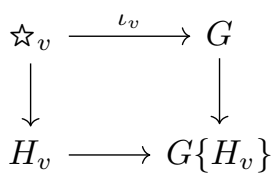

in $\widetilde{\mathbf{U}}$.

Lemma 3.18. Let $Y$ be a $\mathbf{U}$-presheaf satisfying the Segal condition and let $M$ be the modular operad associated to $Y$ (Remark 3.10 and Definition 3.17). There is a canonical bijection

$$
Y_{G} \stackrel{f_{G}}{\longrightarrow}(N M)_{G}
$$

for every $G \in \mathbf{U}$. The map $f$ is a morphism of $\widehat{\mathbf{U}}$.

Proof. By definition of $M$, there exist bijections

$$
Y_{\uparrow} \stackrel{\cong}{\longrightarrow}(N M)_{\uparrow}=\operatorname{ModOp}(\mathrm{U}[\uparrow], M)
$$

and

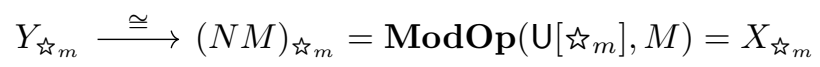

which are compatible with the embeddings $\uparrow \rightarrow \xi_{m}$.

For a graph $G$ with at least one internal edge, the map $f_{G}$ is given by the composition

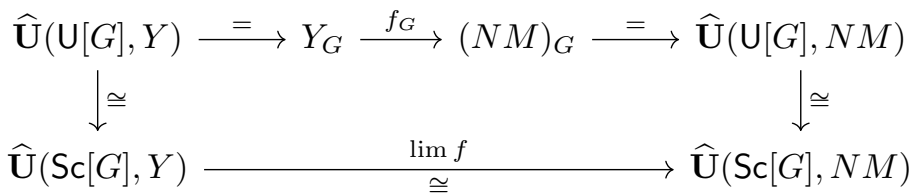

where the vertical arrows are the Segal maps of Definition 3.4. and the two bijections in the top come from the Yoneda Lemma. The bottom isomorphism follows from the first paragraph, and the Segal map for the nerve $N M$ is a bijection by Lemma 3.8 .

Proof of Theorem 3.6. We've already shown that the nerve functor is fully faithful in Proposition 3.9. Satisfying the Segal condition is preserved by isomorphism, so one direction follows immediately from Lemma 3.8. The other direction is Lemma 3.18

We already know (by [HRY20, Theorem 4.12]) that the category of Segal Upresheaves is equivalent to the category of Segal U-presheaves. This latter category is equivalent to ModOp, but we can say a bit more. The functor $\mathbf{U} \rightarrow \operatorname{ModOp}$ from $\$ 2.2$ extends to $\widetilde{\mathbf{U}} \rightarrow$ ModOp by sending a nodeless loop, with $\operatorname{arc}$ set $A$, to the initial object of $\mathbf{M o d O} \mathbf{p}_{A}$. Note that this extended functor is not injective on isomorphism classes of objects, as it was in Proposition 2.25. the exceptional edge maps to the same modular operad as in Example 2.19.

Remark 3.19. The analogue of Theorem 3.6 holds for the functor $\widetilde{\mathbf{U}} \rightarrow$ ModOp. Temporarily write $N^{\prime}$ for the associated functor from ModOp to $\widetilde{\mathbf{U}}$-presheaves. We have $N=\iota^{*} N^{\prime}$. As any nodeless loop and the exceptional edge produce the same modular operad, we can conclude (using also Lemma 3.8) that $N^{\prime}(P)$ is Segal. This 
also shows that $N^{\prime}=\iota_{*} N$ by [HRY20, Theorem 4.12] (or more precisely, that the unit $N^{\prime}(Q) \rightarrow \iota_{*} \iota^{*} N^{\prime}(Q)$ is an isomorphism for each $\left.Q\right)$. We have

$$
\begin{aligned}
\operatorname{hom}\left(N^{\prime}(P), N^{\prime}(Q)\right) & =\operatorname{hom}\left(N^{\prime}(P), \iota_{*} \iota^{*} N^{\prime}(Q)\right) \\
& =\operatorname{hom}\left(\iota^{*} N^{\prime}(P), \iota^{*} N^{\prime}(Q)\right) \\
& =\operatorname{hom}(N(P), N(Q)) \\
& =\operatorname{ModOp}(P, Q) .
\end{aligned}
$$

so we see that $N^{\prime}$ is fully-faithful. Finally, the construction in 3.1 was already phrased in terms of Segal $\widetilde{\mathbf{U}}$-presheaves and the proof of Lemma 3.18 holds for $N^{\prime}$.

Remark 3.20. There is a category of colored cyclic operads (see DCH18, Shu20]) Cyc, which can be defined using monads as in Section 2.1, except only using simplyconnected graphs with nonempty boundary. Let $\mathbf{U}_{\text {cyc }}$ denote the full subcategory of $\mathbf{U}$ on the simply-connected graphs with nonempty boundary [HRY20, Section 5]. There is a forgetful functor ModOp $\rightarrow \mathbf{C y c}$, and the composite

$$
\mathbf{U}_{\mathrm{cyc}} \rightarrow \mathrm{U} \rightarrow \text { ModOp } \rightarrow \text { Cyc }
$$

(where the middle functor is from Proposition 2.25) is fully-faithful and injective on objects. The reader should contrast this situation with Example 2.26 and HRY19, Example 5.7]. We expect that $\mathbf{U}_{\mathrm{cyc}} \rightarrow \mathbf{C y c}$ is thus amenable to the techniques of Web07 and BMW12]; in particular, the analogue of Theorem 3.6 may formally follow from Weber theory.

\section{The Nerve TheOREM OF Joyal And Kock}

In Section 2.2 we indicated how each graph determines a modular operad and that this constitutes a functor $J: \mathbf{U} \rightarrow$ ModOp. In fact, this factors as

$$
\mathrm{U} \stackrel{\iota}{\rightarrow} \mathbf{G r} \stackrel{I}{\rightarrow} \text { ModOp, }
$$

where $\mathbf{G r}$ (previously seen in Remark 1.8) is the category of Feynman graphs of Joyal and Kock. The latter functor in this composition appeared in JK11, though its existence shouldn't be surprising: Remark 2.18 extends to étale maps, that is, every étale map $f: K \rightarrow G$ determines an element of $\langle G\rangle\left(\widetilde{\partial}(K),\left.f\right|_{\Im(K)}\right)^{5}$

On page 112 of [JK11, the following theorem is announced. Details were promised in a forthcoming manuscript, which has not appeared in the intervening eight years.

Theorem 4.1 (Joyal and Kock). The functor I : Gr $\rightarrow$ ModOp induces a fully faithful functor

$$
N_{J K}: \text { ModOp } \rightarrow \widehat{\mathbf{G r}}
$$

where $N_{J K}(P)=\operatorname{ModOp}(I(-), P)$. The essential image of $N_{J K}$ is characterized by the Segal condition.

The reader should note the similarities between this theorem and our Theorem 3.6 The purpose of the present section is to show how our nerve theorem implies that of Joyal and Kock. This provides an independent proof of this theorem (whose original proof was never made public) using alternative techniques. We would also like to point the reader to the thesis of Sophie Raynor [Ray18, which takes a different approach to prove a related nerve theorem for modular operads.

\footnotetext{
${ }^{5}$ Note, though, that $\left.f\right|_{\Im(K)}$ need not be injective when $f$ is not an embedding. This implies that we cannot make the same choices we made in Remark 2.18 for étale maps.
} 
The functor $\iota: \mathbf{U} \rightarrow \mathbf{G r}$ induces adjunctions

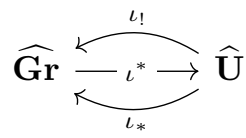

where $\iota_{*}$ (resp. $\iota_{\text {! }}$ ) is given by right (resp. left) Kan extension along $\iota^{\mathrm{op}}: \mathbf{U}^{\mathrm{op}} \rightarrow \mathbf{G r}^{\mathrm{op}}$. The functor $\iota$ i is the left adjoint of $\iota^{*}$, which in turn is the left adjoint of $\iota_{*}$.

The categories $\mathbf{U}$ and $\mathbf{G r}$ have the same set of objects, and by the Yoneda lemma the object $\iota ! \mathrm{U}[G]$ is the representable object $\mathbf{G r}(-, G)$. One can define the Segal condition via Segal cores exactly as in this paper, and then one would see that $\iota ! \operatorname{Sc}[G] \rightarrow \iota ! \mathrm{U}[G]$ is the $\mathbf{G r}$-analogue of the Segal core inclusion since $\iota$ ! is cocontinuous. As the diagram

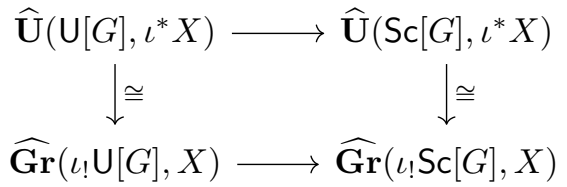

is commutative for every $G$, we see that an object $X \in \widehat{\mathbf{G r}}$ satisfies the Segal condition if and only if $\iota^{*} X \in \widehat{\mathbf{U}}$ satisfies the Segal condition.

Lemma 4.2. If $P$ is a modular operad, then $N_{J K}(P) \in \widehat{\mathbf{G r}}$ satisfies the Segal condition.

Proof. Consider the diagram 12 for $X=N_{J K}(P)$ and for an arbitrary graph $G$. Since $N(P) \cong \iota^{*} N_{J K}(P)$, we know that the top map is an isomorphism by Lemma 3.8 hence so is the bottom map.

Lemma 4.3. The functor $N_{J K}:$ ModOp $\rightarrow \widehat{\mathbf{G r}}$ is fully faithful. Furthermore, there is a natural isomorphism of functors $N_{J K} \cong \iota_{*} N$.

Proof. Consider the composition of functors

$$
\mathbf{U} \stackrel{\iota}{\rightarrow} \mathbf{G r} \stackrel{I}{\rightarrow} \text { ModOp. }
$$

The following two functors are fully faithful:

- the functor $I: \mathbf{G r} \rightarrow \operatorname{ModOp}$ (see [JK11, §6]), and

- the functor $N(-)=\operatorname{ModOp}(I \iota,-)$ from ModOp to $\widehat{\mathbf{U}}$ (Proposition 3.9. Both statements of the lemma are then consequences of [LP08, Proposition 1.1].

We say that a graph is elementary if it is isomorphic to either the exceptional edge $\uparrow$ or to a star $\boldsymbol{\imath}_{n}$.

For the proof of the following lemma, it is convenient to utilize the pointwise description of right Kan extension (see, for instance, Theorem 1 of [ML98, X.3]). Recall that if $Y \in \widehat{\mathbf{U}}$, then

$$
\left(\iota_{*} Y\right)_{G}=\lim _{\substack{G \downarrow \iota^{\mathrm{op}} \\ G \rightarrow H}} Y_{H}
$$

where $G \downarrow \iota^{\text {op }}$ has objects $\mathbf{G r}^{\mathrm{op}}(G, H)=\mathbf{G r}(H, G)$ as $H$ varies, and morphisms from $G \rightarrow H$ to $G \rightarrow H^{\prime}$ are those maps in $\mathbf{U}^{\text {op }}\left(H, H^{\prime}\right)=\mathbf{U}\left(H^{\prime}, H\right)$ making the 
diagram

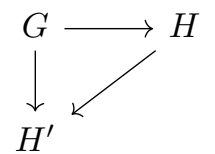

commute in $\mathbf{G r}^{\text {op }}$.

Lemma 4.4. The counit $\iota^{*} \iota_{*} \Rightarrow \mathrm{id}$ of the adjunction $\iota^{*} \dashv \iota_{*}$ is an isomorphism on each elementary graph. In other words, for each presheaf $Y \in \widehat{\mathbf{U}}$ and each elementary graph $K$, we have $\left(\iota_{*} Y\right)_{K} \cong Y_{K}$. Furthermore, if $K$ and $K^{\prime}$ are elementary graphs and $f \in \mathbf{G r}\left(K, K^{\prime}\right)$, then $f \in \mathbf{U}\left(K, K^{\prime}\right)$ and $\left(\iota_{*} Y\right)_{f} \cong Y_{f}$.

Proof. If $K$ is an elementary graph, then $\mathbf{G r}(H, K)=\mathbf{U}(H, K)$. This implies that the object $\mathrm{id}_{K}^{\mathrm{op}}$ is initial in the category $K \downarrow \iota^{\mathrm{op}}$. Thus the inclusion $\left\{\operatorname{id}_{K}^{\mathrm{op}}\right\} \hookrightarrow K \downarrow \iota^{\mathrm{op}}$ induces an isomorphism

$$
\left(\iota_{*} Y\right)_{K}=\lim _{K \downarrow \iota^{\text {op }}} Y_{H} \rightarrow \lim _{\left\{\mathrm{id}_{K}^{\text {op }}\right\}} Y_{K}=Y_{K}
$$

This proves the first statement. The final sentence of the lemma follows immediately from naturality and the fact that $\mathbf{G r}\left(K, K^{\prime}\right)=\mathbf{U}\left(K, K^{\prime}\right)$.

Lemma 4.5. If $X \in \widehat{\mathbf{G r}}$ satisfies the Segal condition, then $X \cong N_{J K}(P)$ for some $P \in$ ModOp.

Proof. As mentioned above, $X$ satisfying the Segal condition is equivalent to $\iota^{*} X$ satisfying the Segal condition by the square $(12)$. Since $\iota^{*} X \in \widehat{\mathbf{U}}$ is Segal, there exists a $P \in \operatorname{ModOp}$ and an isomorphism $\iota^{*} X \stackrel{\cong}{\longrightarrow} N(P)$ by Theorem 3.6. We thus have an isomorphism

$$
\iota_{*} \iota^{*} X \stackrel{\cong}{\longrightarrow} \iota_{*} N(P)
$$

after right Kan extension; by Lemma 4.3 we know $N_{J K}(P) \cong \iota_{*} N(P)$.

Write $f$ for the composite

$$
X \rightarrow \iota_{*} \iota^{*} X \stackrel{\cong}{\longrightarrow} N_{J K}(P)
$$

where the first map is the unit of the adjunction $\iota^{*} \dashv \iota_{*}$. We claim that for each elementary graph $K$, the first map $X_{K} \rightarrow\left(\iota_{*} \iota^{*} X\right)_{K}$ is an isomorphism. Indeed, this map is the first map in the composite

$$
\left(\iota^{*} X\right)_{K} \rightarrow\left(\iota^{*} \iota_{*} \iota^{*} X\right)_{K} \rightarrow\left(\iota^{*} X\right)_{K}
$$

which is the identity function by one of the triangle identities for an adjunction (see Theorem 1(ii)(8) in [ML98, XI.1, p.82]). We showed that the second map $\left(\iota^{*} \iota_{*} \iota^{*} X\right)_{K} \rightarrow\left(\iota^{*} X\right)_{K}$ was an isomorphism in Lemma 4.4 so the claim follows.

We now know that morphism $f$ of $\widehat{\mathbf{G r}}$ has the property that $f_{K}: X_{K} \rightarrow N_{J K}(P)_{K}$ is an isomorphism for every elementary graph $K$. Since both $X$ and $N_{J K}(P)$ are Segal, this implies that $f$ is an isomorphism.

Proof of Theorem 4.1. By Lemma 4.3, we know that $N_{J K}$ is fully faithful. As satisfying the Segal condition is invariant under isomorphism, we know by Lemma 4.2 that every $X$ in the essential image of $N_{J K}$ satisfies the Segal condition. Lemma 4.5 provides the reverse containment. 
In this section we showed that Theorem 3.6 implies Theorem 4.1. This implication was mostly formal, relying on that fact that $\iota$ is a bijection on objects, the coincidence of the subcategories of elementary graphs, and fully faithfulness of $I: \mathbf{G r} \rightarrow$ ModOp. As there is no backwards version of Proposition 1.1 of [LP08, it seems unlikely that one can recover Theorem 3.6 from Theorem 4.1

\section{REFERENCES}

[BB17] M. A. Batanin and C. Berger, Homotopy theory for algebras over polynomial monads, Theory Appl. Categ. 32 (2017), Paper No. 6, 148-253. MR 3607212

[BM11] Clemens Berger and Ieke Moerdijk, On an extension of the notion of Reedy category, Math. Z. 269 (2011), no. 3-4, 977-1004. MR 2860274

[BMW12] Clemens Berger, Paul-André Melliès, and Mark Weber, Monads with arities and their associated theories, J. Pure Appl. Algebra 216 (2012), no. 8-9, 2029-2048. MR 2925893

[CGR14] Eugenia Cheng, Nick Gurski, and Emily Riehl, Cyclic multicategories, multivariable adjunctions and mates, J. K-Theory 13 (2014), no. 2, 337-396. MR 3189430

[CH19] Hongyi Chu and Rune Haugseng, Homotopy-coherent algebra via Segal conditions, Preprint, arXiv:1907.03977v1 [math.AT], 2019.

[CM13] Denis-Charles Cisinski and Ieke Moerdijk, Dendroidal Segal spaces and $\infty$-operads, J. Topol. 6 (2013), no. 3, 675-704.

[DCH18] Gabriel C. Drummond-Cole and Philip Hackney, Dwyer-Kan homotopy theory for cyclic operads, Preprint, arXiv:1809.06322v1 [math.AT], 2018.

[DCH19] _ A criterion for existence of right-induced model structures, Bull. Lond. Math. Soc. 51 (2019), no. 2, 309-326.

[Dou17] Martin Doubek, The modular envelope of the cyclic operad Ass, Appl. Categ. Structures 25 (2017), no. 6, 1187-1198. MR 3720407

[Gia13] Jeffrey Giansiracusa, Moduli spaces and modular operads, Morfismos 17 (2013), no. 2, 101-125.

[GK98] E. Getzler and M. M. Kapranov, Modular operads, Compositio Math. 110 (1998), no. 1, 65-126. MR 1601666

[HRY15] Philip Hackney, Marcy Robertson, and Donald Yau, Infinity properads and infinity wheeled properads, Lecture Notes in Mathematics, vol. 2147, Springer, Cham, 2015. MR 3408444

[HRY17] _ Shrinkability, relative left properness, and derived based change, New York J. Math. 23 (2017), 83-117. MR 3611076

[HRY18] - On factorizations of graphical maps, Homology Homotopy Appl. 20 (2018), no. 2, 217-238. MR 3812464

[HRY19] , Higher cyclic operads, Algebr. Geom. Topol. 19 (2019), no. 2, 863-940. MR 3924179

[HRY20] _ A graphical category for higher modular operads, Adv. Math. 365 (2020), 107044. MR 4064770

[HVZ10] Eric Harrelson, Alexander A. Voronov, and J. Javier Zúñiga, Open-closed moduli spaces and related algebraic structures, Lett. Math. Phys. 94 (2010), no. 1, 1-26. MR 2720252

[Jac99] Bart Jacobs, Categorical Logic and Type Theory, Studies in Logic and the Foundations of Mathematics, vol. 141, North-Holland Publishing Co., Amsterdam, 1999. MR 1674451

[JK11] André Joyal and Joachim Kock, Feynman graphs, and nerve theorem for compact symmetric multicategories (extended abstract), Electronic Notes in Theoretical Computer Science 270 (2011), no. 2, 105-113.

[JS91] André Joyal and Ross Street, The geometry of tensor calculus. I, Adv. Math. 88 (1991), no. 1, 55-112. MR 1113284

[Koc11] Joachim Kock, Polynomial functors and trees, Int. Math. Res. Not. IMRN (2011), no. 3, 609-673. MR 2764874

[Koc16] - Graphs, hypergraphs, and properads, Collect. Math. 67 (2016), no. 2, 155-190. MR 3484016

[KW17] Ralph M. Kaufmann and Benjamin C. Ward, Feynman categories, vol. 387, Soc. Math. France, 2017. 
[LP08] Stephen Lack and Simona Paoli, 2-nerves for bicategories, K-Theory 38 (2008), no. 2, 153-175. MR 2366560

[Mar08] Martin Markl, Operads and PROPs, Handbook of algebra. Vol. 5, Handb. Algebr., vol. 5, Elsevier/North-Holland, Amsterdam, 2008, pp. 87-140. MR 2523450

[Mar16] , Modular envelopes, OSFT and nonsymmetric (non- $\Sigma$ ) modular operads, J. Noncommut. Geom. 10 (2016), no. 2, 775-809. MR 3519052

[ML98] Saunders Mac Lane, Categories for the working mathematician, second ed., Graduate Texts in Mathematics, vol. 5, Springer-Verlag, New York, 1998 . MR 1712872 (2001j:18001)

[MW07] Ieke Moerdijk and Ittay Weiss, Dendroidal sets, Algebr. Geom. Topol. 7 (2007), 14411470. MR 2366165 (2009d:55014)

[MW09] - On inner Kan complexes in the category of dendroidal sets, Adv. Math. 221 (2009), no. 2, 343-389. MR 2508925 (2010a:55021)

[Pet13] Dan Petersen, The operad structure of admissible G-covers, Algebra Number Theory 7 (2013), no. 8, 1953-1975. MR 3134040

[Ray18] Sophie Raynor, Compact symmetric multicategories and the problem of loops, 2018, PhD. thesis, University of Aberdeen.

[Ray19] , A distributive law for compact symmetric multicategories, Preprint, arXiv:1911.05914v1 [math.CT], 2019.

[Shu20] Michael Shulman, The 2-Chu-Dialectica construction and the polycategory of multivariable adjunctions, Theory Appl. Categ. 35 (2020), 89-136.

[Web07] Mark Weber, Familial 2-functors and parametric right adjoints, Theory Appl. Categ. 18 (2007), No. 22, 665-732. MR 2369114

[YJ15] Donald Yau and Mark W. Johnson, A Foundation for PROPs, Algebras, and Modules, Mathematical Surveys and Monographs, vol. 203, American Mathematical Society, Providence, RI, 2015. MR 3329226

Department of Mathematics, University of Louisiana at Lafayette, Lafayette, LA 70504-3568 USA

E-mail address: philip@phck.net

$U R L:$ http://phck.net

School of Mathematics and Statistics, The University of Melbourne, Melbourne, Victoria, Australia

E-mail address: marcy.robertson@unimelb.edu.au

Department of Mathematics, The Ohio State University at Newark, Newark, OH, USA

E-mail address: dyau@math.ohio-state.edu 